\title{
ISOCAM observations of the very deep IRAS 60 micron sample in the NEP region ${ }^{\star}$
}

\section{The data}

\author{
H. Aussel ${ }^{1}$, D. Coia ${ }^{2}$, P. Mazzei ${ }^{1}$, G. De Zotti ${ }^{1}$, and A. Franceschini ${ }^{2}$ \\ 1 Osservatorio Astronomico di Padova, Vicolo dell'Osservatorio 5, I-35122 Padova, Italy \\ 2 Dipartimento di Astronomia dell'Università di Padova, Vicolo dell'Osservatorio 5, I-35122 Padova, Italy
}

Received September 10; accepted October 26, 1999

\begin{abstract}
We present the results of ISO observations with the CAM LW3 filter, centered at $\lambda_{\text {eff }}=14.3 \mu \mathrm{m}$, of 94 out of the 98 galaxies comprising the complete $60 \mu \mathrm{m}$ IRAS deep survey (IDS) sample in the north ecliptic polar region. In addition, we observed a source detected by IRAS at $25 \mu \mathrm{m}$ and found to have particularly interesting properties. Altogether, 106 sources were detected with a signal to noise ratio $\geq 3$ and 69 with $S / N \geq 5$ in the $3.2^{\prime} \times 3.2^{\prime}$ fields centered on the nominal positions of IRAS sources. Sixty-five $\geq 3 \sigma$ detections ( 49 of which at $\geq 5 \sigma$ ) are likely identifications of IRAS sources. Ten additional IRAS sources have possible $\geq 3 \sigma$ ISOCAM counterparts. In 6 further cases, signals at the $2-3 \sigma$ level were detected close to the IRAS position. Indications that IRAS sources might actually be multiple (source confusion) were found in 4 IDS fields. On the whole, we confirm the reality of 69 to $90 \%$ of IDS sources. Appropriate statistical corrections for the bias affecting faint flux estimates were applied to ISOCAM data. Ten serendipitous sources were detected at $\geq 5 \sigma$, with $S(14.3 \mu \mathrm{m}) \geq 3.5 \mathrm{mJy}$. The corresponding areal density is consistent with that found in previous surveys. Finding charts for all observed fields are given.
\end{abstract}

Key words: surveys — galaxies: photometry — galaxies: starburst - infrared: galaxies

Send offprint requests to: G. De Zotti,

e-mail: dezotti@pd.astro.it

* Based on observations with the Infrared Space Observatory (ISO). ISO is an ESA project with instruments funded by ESA Member States (especially by the PI countries: France, Germany, The Netherlands and the United Kingdom) and with participation of ISAS and NASA.

\section{Introduction}

IRAS scanned the north ecliptic polar region (NEPR) over 1000 times from all directions. Hacking (1987) coadded these scans, representing more than 20 hours of integration time, and obtained point source filtered maps used by Hacking \& Houck (1987, henceforth HH87) to compile the deepest FIR samples available before the advent of ISO surveys. The $60 \mu \mathrm{m}$ sample (henceforth referred to as the IRAS Deep Survey sample, IDS), probably constituted entirely of galaxies, has long been the most crucial piece of information on which numerous studies (see, e.g.: Ashby et al. 1996; Hacking et al. 1987; Oliver et al. 1992; Treyer \& Silk 1993; Franceschini et al. 1994) of the far-IR evolution of galaxies relied. It is comprised of 98 sources (plus the planetary nebula NGC 6543) with $60 \mu \mathrm{m}$ fluxes $\geq 50 \mathrm{mJy}$ in an area of 6.25 square degrees. Most (77) sources also have $100 \mu \mathrm{m}$ fluxes (although 25 of these are rather uncertain); 17 were detected at $25 \mu \mathrm{m}$, but only 5 at $12 \mu \mathrm{m}$. Ashby et al. (1996) took optical spectra of 76 tentative IDS identifications at the Palomar $5 \mathrm{~m}$ telescope.

We have carried out ISO observations with the CAM LW3 filter (range $12-18 \mu \mathrm{m}, \lambda_{\text {eff }}=14.3 \mu \mathrm{m}$ ) of 94 IDS sources plus a source detected by IRAS at $25 \mu \mathrm{m}$ and found to have particularly interesting properties.

The scientific rationale of these observations is severalfold. First, we aimed at assessing the reliability of faint $60 \mu \mathrm{m}$ sources themselves and, hence, of their counts below $100 \mathrm{mJy}$. As pointed out by HH87, the flux uncertainties of their faintest sources is $\simeq 20 \mathrm{mJy}$ so that a $50 \mathrm{mJy}$ sources is a $2.5 \sigma$ detection. At this low $S / N$ level, the interpretation of the data requires a careful quantification of completeness, reliability and measurement biases. Spurious sources may be produced by the significant cirrus contamination affecting the field. Also, due to the limited angular resolution of the survey, some faint IRAS "sources" may actually be multiple systems. In fact, there 
is a considerable uncertainty on the faint end of $60 \mu \mathrm{m}$ counts, different studies yielding estimates differing by factors up to $\simeq 2$ (HH87, Gregorich et al. 1995; Bertin et al. 1997).

Second, ISOCAM observations allow a significant improvement of the positional accuracy of sources and a corresponding improvement of the reliability of optical identifications. An accurate location is essential since some of these sources may be optically faint either because of a high obscuration by dust or because they are very distant. But the large surface density of optically faint sources makes the identification process very uncertain unless the error box is correspondingly small. This is particularly critical to correctly trace cosmological evolution. For example, some models predict that a few IDS sources may be dust enshrouded galaxies at substantial redshifts; but these sources may easily be misidentified with brighter galaxies in the field.

Third, our measurements establish a direct link between IRAS and ISOCAM counts. The former cover fluxes up to two orders of magnitude brighter than the shallow surveys in the LW3 filter (Rowan-Robinson et al. 1999; Désert et al. 1999) and are therefore a very important complement to the latter.

Fourth, combining ISOCAM and IRAS data we can analyze the far-IR spectral energy distribution (SED) of a relatively large sample of far-IR selected galaxies, covering several Gyr in lookback time. It will be interesting to see, for example, if the cosmological evolution clearly indicated by the $60 \mu \mathrm{m}$ counts is somehow reflected in an evolution of the SED.

Fifth, from the bivariate $60 \mu \mathrm{m} / 14.3 \mu \mathrm{m}$ luminosity distribution and the $60 \mu \mathrm{m}$ local luminosity function, we may derive an estimate of the poorly known $14.3 \mu \mathrm{m}$ local luminosity function.

In this paper, the first of a series, we present and briefly discuss ISOCAM data. In a second paper we will compare ISOCAM fluxes with IRAS and radio data and discuss the spectral energy distribution of sources in the far-IR to radio region. The third paper will deal with optical identifications, spectroscopy and photometry.

\section{Observations}

We have observed the entire IDS except for the planetary nebula NGC 6543, the bright galaxy NGC 6552 $(18001+6636)$, which has a good IRAS measurement of the $12 \mu \mathrm{m}$ flux, and the last three sources in the IDS list $(18111+6636,18115+6706$, and $18116+6536)$ because of lack of observing time. On the whole, we got data for 94 IDS fields. In addition, we have observed the $25 \mu \mathrm{m}$ detection (object 2-16 in Table 5 of HH87) with very unusual far-IR colours which turned out (Ashby \& Hacking, private communication) to be a very high luminosity, distant post-starburst galaxy.
Observations were performed on 19 and 20 December 1996 and on 13 February 1997 via the CAM03 (beamswitching) AOT (Astronomical Observation Template: the instrument observing mode) with the LW3 filter, a pixel field of view of $6^{\prime \prime}$ (since the detector comprises an array of $32 \times 32$ pixels, its field of view is then of $3.2^{\prime} \times 3.2^{\prime}$ ), and a throw of $3^{\prime}$ at constant declination. The typical number of exposures to stabilize the detectors to the sky background before each observation was $N_{\text {stab }}=60$ and the number of exposures used to measure the source flux was typically $N_{\exp }=22$. An equal number of exposures was taken on sources and on reference fields (one per source); because of the detector stabilization problem, exposures on the reference field were taken after those on the source were completed (rather than taking on/off source exposures in sequence). The integration time per exposure was of 2.1 seconds. The exposures were more numerous for fields observed at the beginning of each set of concatenated observations, to allow sufficient time for instruments to stabilize in the new configuration. Observations were completed in a single cycle of source-reference field pointings.

Table 1 gives the observing log. Columns 1 and 2 list the source names according to Table 5 of HH87 and to the IRAS Faint Source Survey (FSS; Moshir et al. 1992). Columns 3 and 4 give the equatorial coordinates, for the equinox 2000, of the centers of the observed $3.2^{\prime} \times 3.2^{\prime}$ fields; these correspond to the coordinates listed by Ashby et al. (1996), which coincide with the coordinates of IRAS sources given by HH87 except for the 9 sources (3-02, 304, 3-08, 3-22, 3-35, 3-43, 3-74, 3-82, 3-89) for which the coordinates of optical identifications are provided. Column 5 gives the total time spent on each target, Col. 6 the observation dates, and Col. 7 the so called "TDTOSN" numbers identifying each observation in the ISO data archive.

\section{Data analysis}

Data were reduced using the Cam Interactive Analysis (CIA) package (Ott et al. 1998), release 3.0, jointly developed by the ESA Astrophysics Division and the ISOCAM Consortium. Dark current subtraction and calculation of the flat-field response for each pixel were done using the software provided by ESA together with the data files. The Multi-resolution Median Transform (MMT) method (Starck et al. 1996), which looks for signals on timescales shorter than the total integration time per frame, was used to identify glitches. The method proved to be robust and to work well even with unstabilized data. However, it is not designed to correct the long term gain variations of the pixel occurring after some impact of cosmic rays, because this phenomenon has a timescale longer than those examined by the MMT. This would cause spurious signals to show up after substraction of the reference image from the on-source one. To overcome this problem, the time history of each pixel after major glitches was examined and 
the readouts showing signs of cosmic-induced drifts were masked by hand.

It must also be taken into account that, when its illumination changes, the ISOCAM LW detector is affected by transient effects which may either cause a photometric error or the appearance of "ghost" sources after a bright source has been observed. Corrections for transients have been computed using the method by Abergel et al. (1996; see also Starck et al. 1999).

Source fluxes were determined by means of direct aperture photometry on the maps. Fluxes were measured within radii of $6^{\prime \prime}, 9^{\prime \prime}$, and $12^{\prime \prime}$; the highest value was adopted. An aperture correction was applied; it was computed using the average ratio between fluxes measured in the same aperture for a theoretical point spread function (Okumura 1997) and its integrated flux on the whole array. We converted the Analogic to Digital Units (ADU) per gain and per second $(\mathrm{ADU} / \mathrm{G} / \mathrm{s})$ of the map in $\mathrm{mJy}$, using the conversion factor given with the October '98 release of CIA, that is: $1 \mathrm{ADU} / \mathrm{G} / \mathrm{s}=0.5068 \mathrm{mJy}$. In a few cases it was possible to check the flux estimate by measuring the negative imprint produced by beam-switching. Fluxes measured in this way were always in good agreement with the those measured on the positive source, even in the case of sources below the $3 \sigma$ threshold. For this reason we have included in Table 2 also $2 \sigma$ detections.

Astrometry of ISOCAM images, as computed from the pointing information provided by the satellite, suffers from two sources of uncertainties. The first one comes from ISO pointing accuracy. When acquiring a target, the absolute pointing error has been measured to be about 2 .'5 $(2 \sigma)$ during the period when most observations have been done (Leech 1998). The second source of astrometric uncertainty is the so called "lens jitter" of the ISOCAM instrument (Siebenmorgen et al. 1999): the position of the lens wheel in front of the detector array is not fully reproducible due to a play in the wheel. This induces a shift of the images that can reach 2 to 3 pixels (i.e. 12 to 18 arcsec) between two extreme positions of the wheel. Therefore, the astrometry of a given image is precise to $20^{\prime \prime}$.

This precision is not sufficient for our purpose, but a much better astrometry can be acheived by taking advantage of the way our observations have been scheduled. The "TDTOSN" numbers given in the last column of Table 1 consist of the revolution number when the observation was taken (first 3 digits), of the sequence number of the observation during the revolution or TDT number (next 3 digits), and of the sequence number in this sequence or OSN number (last 2 digits). Our observations were taken during 4 revolutions $(398,399,400$ and 455). During a revolution, the observations were performed on consecutive sequences of concatenated chains of beam-switch. During a concatenated chain of observations, identified by the same revolution number and TDT number, the optical configuration of the camera did not change. Hence, if we can measure the pointing offset due to the lens jitter in one of the field observed during a chain of concatenated observations, the same offset will also apply to all the observations of the chain. Between two consecutive concatenated chains of beam-switches, the camera goes back to the "standby" mode, that is to the 6 " pixel field of view lens and to the LW2 filter. Since we are using the same lens, the lens wheel will not move between a sequence of consecutive TDT number. Therefore, if we can manage to measure the pointing offset in one of the fields of a given revolution, we can safely apply the same offset to all observations performed during that revolution.

To measure the offset we have searched for field where the optical counterpart of the ISOCAM source is identified without any doubt. Whenever possible, we have chosen fields where more than one source is detected, and where these sources are point like. Unfortunately, we only have a few fields matching these criteria, and all in revolutions 399 and 400. We have therefore used also various fields where there is only one CAM source, but with a clear-cut identification (source detected in radio by Hacking et al. (1989) or only one possible candidate). The positions of sources on the map were derived by fitting a PSF computed from the model of Okumura (1997) for high signalto-noise sources, or using the brightest pixel center. The distortion of the map was taken into account using the measurements by Aussel et al. (1999). Positions of the optical counterparts were measured on the Digitized Sky Survey (DSS; available at stdatu.stsci.edu/dss) ${ }^{1}$.

For observations taken during revolution 398 we have used sources 3-09 and 3-11 having unambiguous nearby optical counterparts which are also radio sources (Hacking et al. 1989). The source in the field 3-15 would also satisfy our criteria but it is slightly extended, so that its positional uncertainty is larger. The offset between ISOCAM and optical positions is $\Delta \alpha=-12^{\prime \prime}, \Delta \delta=-2^{\prime \prime}$.

For observations taken during revolution 399 we have used the sources A, B and C in the field 3-19 and sources $\mathrm{B}, \mathrm{C}$, and D in the field 3-26 (source A looks extended). The derived offset is $\Delta \alpha=-21^{\prime \prime}, \Delta \delta=3^{\prime \prime}$. We found

1 The DSS was produced at the Space Telescope Science Institute under US Government grant NAG W-2166. The images of this survey are based on photographic data obtained using the Oschin Schmidt Telescope on Palomar Mountain. The plates were processed into the present compressed digital form with the permission of this institution. The National Geographic Society - Palomar Observatory Sky Atlas (POSS-I) was made by the California Institute of Technology with grants from the National Geographic Society. The Second Palomar Observatory Sky Survey (POSS-II) was made by the California Institute of Technology with grants from the National Science Foundation, the National Geographic Society, the Sloan Foundation, the Samuel Oschin Foundation, and the Eastman Kodak Corporation. The Oschin Schmidt Telescope is operated by the California Institute of Technology and Palomar Observatory. Supplemental funding for sky-survey work at ST-ScI is provided by the European Southern Observatory. 
a good agreement between offsets derived independently from the two fields, confirming that the lens does not move during the revolution.

For revolution 400 we used source A in the field 3-67 (source B is extended), and sources A and B in the fields 3-79 and 3-80. We obtained an offset of $\Delta \alpha=-18^{\prime \prime}$, $\Delta \delta=+3^{\prime \prime}$.

Finally, for revolution 455, we used the sources $\mathrm{A}$ in the fields 3-02 and 3-41 to find an offset of $\Delta \alpha=-10^{\prime \prime} .5$, $\Delta \delta=-6^{\prime \prime}$.

The ISOCAM source positions given in Table 2 are corrected for offsets as well as for image distortions, which are particularly severe near the field edges. The contours shown in the charts appended to this paper, however, are corrected only for offsets. This is why some sources at the edges of the ISOCAM fields are not well superposed upon their obvious optical counterparts. The final astrometric precision reached after these corrections is of $3 . .25(1 \sigma)$, $1^{\prime \prime} .25$ coming from the absolute pointing accuracy and $3^{\prime \prime}$ from the pixel size for our maps.

\section{Results}

Altogether, 106 sources were detected with a signal to noise ratio $S / N \geq 3$. In addition to those, in Table 2 we list $2-3 \sigma$ signals when there are no ISOCAM detections in the field or when they may be associated to an optical source; these data may be used to derive upper limits to ISOCAM-LW3 fluxes of the corresponding IRAS or optical objects. When no $\geq 2 \sigma$ signal is detected in the field, we give an upper limit equal to 3 times the rms noise of the map at the nominal position of the IRAS source.

In order to assess the reliability of identifications of ISOCAM sources with IRAS sources we have computed, for all ISOCAM sources detected at $\geq 3 \sigma$ (less those in the fields 3-19, 3-26, 3-78 and 3-81, where IRAS fluxes may be affected by substantial confusion effects ${ }^{2}$ ), the mean number of chance objects, $n_{\mathrm{c}}$, closer to the nominal position of the IRAS source and brighter than the candidate (Downes et al. 1996):

$n_{\mathrm{c}}=\pi \Delta_{\text {IRAS-ISO }}^{2} N\left(>S_{14.3 \mu \mathrm{m}}\right)$.

In the relevant flux density range $(2-30 \mathrm{mJy})$, the $14.3 \mu \mathrm{m}$ integral counts of galaxies are accurately described by (see Elbaz et al. 1999; Aussel 1999; Oliver et al. 1998):

$N\left(>S_{14.3 \mu \mathrm{m}}\right) \simeq 4.710^{-2} S_{14.3 \mu \mathrm{m}}^{-1.34}(\mathrm{mJy}) \operatorname{arcmin}^{-2}$.

An optimal method for determining the reliability of an identification has been presented by Sutherland \& Saunders (1992; see also Wolstencroft et al. 1986). The method, however, requires the prior knowledge of the distribution of $14.3 \mu \mathrm{m}$ fluxes which is not available in our case.

\footnotetext{
${ }^{2}$ ISOCAM sources clearly associated with stars were assumed not to significantly contaminate $60 \mu \mathrm{m}$ fluxes.
}

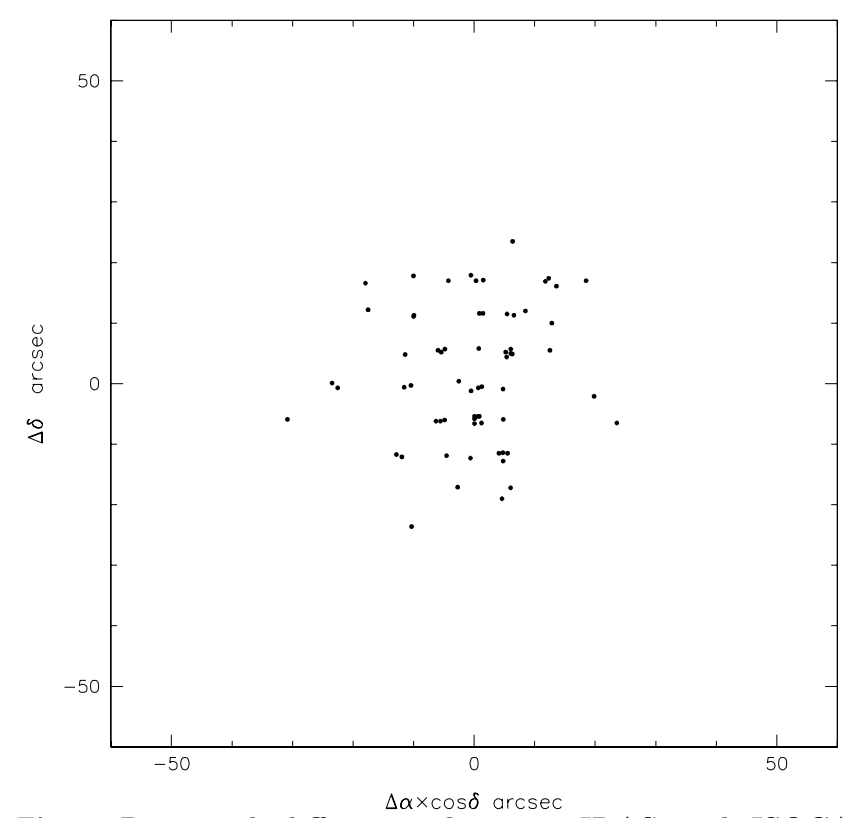

Fig. 1. Positional differences between IRAS and ISOCAM $(\geq 3 \sigma)$ sources

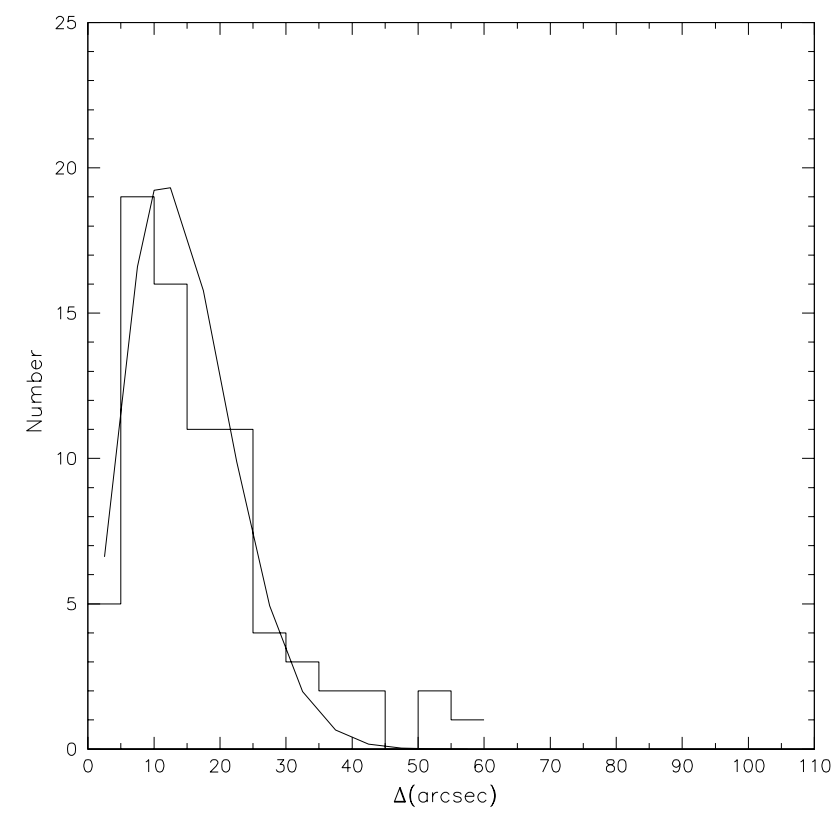

Fig. 2. Distribution of angular separations between IRAS and ISOCAM sources

As shown by Fig. 1, the center of the distribution of differences between IRAS and ISOCAM positions of ISOCAM sources detected at $\geq 3 \sigma$ (excluding the confused fields mentioned above) and with $n_{\mathrm{c}}<510^{-3}$, that we take as likely counterparts to IRAS sources (the expected number of random coincidences in the full sample is 0.5$)$ is not significantly offset from $(0,0)$. We find: $\langle\Delta \alpha \cos \delta\rangle=-0^{\prime \prime} .45 \pm 1^{\prime \prime} .26$ and $\langle\Delta \delta\rangle=1.60 \pm 1.38$. 
The probability that the ISOCAM counterpart has a positional offset $(x, y)$ from the IRAS source is:

$f(x, y) \mathrm{d} x \mathrm{~d} y=\frac{\exp \left[-0.5\left(x / \sigma_{x}\right)^{2}-0.5\left(y / \sigma_{y}\right)^{2}\right]}{2 \pi \sigma_{x} \sigma_{y}} \mathrm{~d} x \mathrm{~d} y$.

The positional error distribution has an approximately circular symmetry (cf. Fig. 1) as expected since the NEPR field was scanned from many different directions (Hacking $\&$ Houck 1987). Then, $\sigma_{x}=\sigma_{y}=\sigma$ and, in polar coordinates:

$f(\Delta) \mathrm{d} \Delta=\frac{\exp \left[-0.5(\Delta / \sigma)^{2}\right]}{\sigma^{2}} \Delta \mathrm{d} \Delta$.

The distribution of positional offsets, $\Delta$, of ISOCAM sources detected at $\geq 3 \sigma$ (excluding confused fields) with respect to IRAS positions can be represented by Eq. (4), with $\sigma=10^{\prime \prime} 2$ (see Fig. 2). For comparison, the positional precision of single pointed IRAS observations is $\sim 5^{\prime \prime}$ in the in-scan direction and $\sim 20^{\prime \prime}-25^{\prime \prime}$ in the cross-scan direction (Hacking \& Houck 1987); since the NEPR field was scanned from many different directions, the final positional error distribution is much more isotropic and typical values, averaged over all directions will be somewhat larger than $\sim 5^{\prime \prime}$. As discussed in the previous section, positional errors of our ISOCAM sources are estimated to be $\simeq 3^{\prime \prime} 25$. Thus the derived rms error of IRAS positions turns out to be $\sim 10^{\prime \prime}$.

As stressed by Hogg \& Turner (1998), flux estimates for faint sources are systematically biased high (in a statistical sense) because in any given observed flux interval there are more sources "brightened" than "dimmed" by measurement errors, simply due to the fact that faint sources are more numerous than bright ones.

If $\beta$ is the slope of integral source counts, the maximum likelihood true flux $S_{\mathrm{ML}}$ is related to the observed flux $S_{\mathrm{o}}$ by (Hogg \& Turner 1998):

$\frac{S_{\mathrm{ML}}}{S_{\mathrm{o}}}=\frac{1}{2}+\frac{1}{2}\left(1-\frac{4 \beta+4}{r^{2}}\right)^{1 / 2}$,

where $r$ is the signal to noise ratio. In the flux density range of interest here $\beta=1.34$ (cf. Eq. (2)); it follows that there is no maximum likelihood value for $r \leq 3.06$.

Figure 3 shows the distribution of corrected fluxes, $S_{\mathrm{ML}}$, of likely counterparts to IRAS sources $\left(n_{\mathrm{c}}<510^{-3}\right)$ detected at $\geq 5 \sigma$.

In addition to sources identified with IRAS targets, we got $10 \geq 5 \sigma$ serendipitous detections with corrected fluxes (see Eq. (5)) $S_{\mathrm{ML}} \geq 3.5 \mathrm{mJy}$. The total surveyed area is of $3 \prime 2 \times 3 ! 2 \times 95=973 \operatorname{arcmin}^{2}$, which, after subtracting the area covered by targets, a few percent, can be rounded to $950 \mathrm{arcmin}^{2}$. About $20 \%$ of pixels are lost because of contamination by cosmic ray hits, leaving a useful area of $\simeq 0.2 \mathrm{deg}^{2}$.

The number of galaxies over this area above $3.5 \mathrm{mJy}$, expected after Eq. (2) is about 6.7. The model by Franceschini et al. (1991) yields $\simeq 810^{-3}{\text { stars } / \text { arcmin }^{2}}^{2}$ in the NEPR region $\left(l=97^{\circ}, b=30^{\circ}\right)$, brighter than

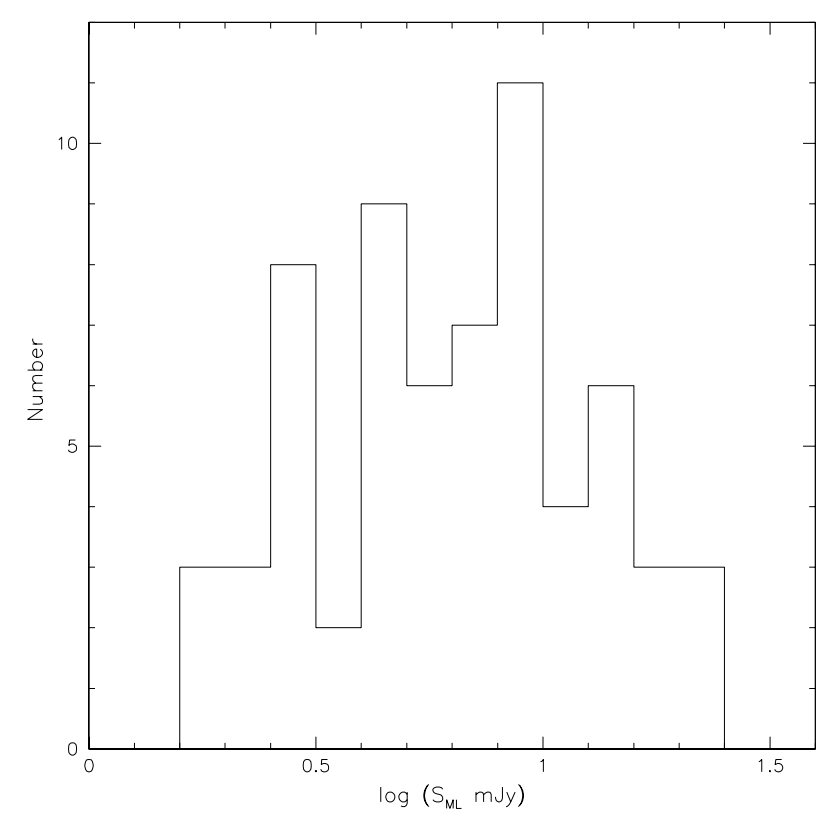

Fig. 3. Distribution of corrected fluxes, $S_{\mathrm{ML}}$, of likely counterparts to IRAS sources $\left(n_{\mathrm{c}}<510^{-3}\right)$ detected at $\geq 5 \sigma$

$5.5 \mathrm{mJy}$ at $12 \mu \mathrm{m}\left(S_{12} \mu \mathrm{m}=5.5 \mathrm{mJy}\right.$ corresponds to $S_{14.3 \mu \mathrm{m}}=3.5 \mathrm{mJy}$ in the case of a Rayleigh-Jeans spectrum peaking at a few $\mu \mathrm{m}$ ), i.e. 7.5 stars in our surveyed area. A slightly higher surface density of stars would be expected based on the results of the deep survey using the LW10 ISOCAM filter, matching the $12 \mu \mathrm{m}$ IRAS filter (Clements et al. 1999). These authors found 13 stars brighter than $0.45 \mathrm{mJy}$ (after correcting fluxes by a factor $1 / 1.25$, according to the prescription in the caption of their Fig. 5, and by the factor given by Eq. (5)) in an area of $0.1 \mathrm{deg}^{2}$, at high Galactic latitute $\left(\langle|b|\rangle \simeq 54^{\circ}\right), 3$ of which are brighter than $5.5 \mathrm{mJy}$. The number of our serendipitous detections is consistent with these results within statistical fluctuations, although a somewhat lower surface density of stars seems to be favoured.

In Table 2 we give: in Col. 1 the target name (HH87), in Cols. 2 and 3 the equatorial coordinates (equinox 2000) of ISOCAM detections, in Col. 4 the position difference (arcsec) between the ISOCAM and IRAS (HH87) sources, in Col. 5 the ISOCAM flux density and its error (mJy), in Col. 6 the maximum likelihood value of the flux density (cf. Eq. (5)) for sources detected at $\geq 5 \sigma$, and in Col. 7 the value of $n_{\mathrm{c}}$.

Appended to this paper are the finding charts for all ISOCAM detections (Fig. 4). The label on top identifies the field (cf. Table 1); the circle encompasses an area of $45^{\prime \prime}$ radius centered on the nominal position of the IRAS source; the contours are isophotes of the ISOCAM sources. The optical charts are from the Digitized Sky Survey. 


\section{Discussion and conclusions}

We have obtained ISOCAM images in the LW3 filter, centered at $\lambda_{\text {eff }}=14.3 \mu \mathrm{m}$, of the $3.2 \times 3.2$ fields containing 94 out of the 98 galaxies comprising the complete $60 \mu \mathrm{m}$ IRAS deep survey (IDS) sample in the north ecliptic polar region. In addition, we observed a source detected by IRAS at $25 \mu \mathrm{m}$ and found to have particularly interesting properties. Charts for all observed fields are given.

We have detected at $\geq 3 \sigma$ the likely $14.3 \mu \mathrm{m}$ counterparts of 65 IDS sources; 10 more possible $\geq 3 \sigma$ identifications are doubtful because of the relatively large difference between nominal ISO and IRAS positions; 6 additional IRAS sources may have been detected at a level between 2 and $3 \sigma$. In 4 further cases, we found indications that IRAS fluxes may be affected by confusion, with two or more sources contributing at a comparable level to the observed $60 \mu \mathrm{m}$ fluxes.

On the whole, our observations confirm the reality of $69-90 \%$ of IDS sources. The 9 IRAS sources with no (even doubtful) $14.3 \mu \mathrm{m}$ counterpart at $\geq 2 \sigma$ are all relatively faint $\left(S_{60 \mu \mathrm{m}}<90 \mathrm{mJy}\right)$; they correspond to $17 \%$ of IDS sources fainter than this limit (our sample comprises 53 such sources). Hacking \& Houck (1987) estimated that the reliability of sources at the survey limit is about $80 \%$; our findings are consistent with their estimate.

Appropriate statistical corrections for the bias affecting faint flux estimates were applied to ISOCAM data.

The areal density of serendipitous sources (stars + galaxies) detected at $\geq 5 \sigma$ in our fields, $N(>3.5 \mathrm{mJy}) \simeq$ $1.310^{-2} \operatorname{arcmin}^{-2}$, is in good agreement with results of ISOCAM surveys at the same frequency.

The substantially improved positional accuracy of ISOCAM, compared to IRAS, makes much easier the identification of optical counterparts, resolving ambiguities pointed out by Ashby et al. (1996). Photometric measurements and redshift determinations of candidate identifications of ISOCAM sources are in progress.

Acknowledgements. We gratefully acknowledge the contributions by Matt Ashby, Perry Hacking and L. Danese during the preparation of the ISO proposal. This work was supported in part by ASI, MURST and the TMR Network "Galaxy Formation" under contract No. ERBFMRX -CT96-0086. The ISOCAM data presented in this paper were analyzed with the CAM Interactive Analysis (CIA) software, a joint development by the ESA Astrophysics Division and the ISOCAM Consortium led by the ISOCAM PI, C. Cesarsky, Direction des Sciences de la Matière, C.E.A., France.

\section{References}

Abergel A., et al., 1996, A\&A 315, L329
Ashby M.L.N., Hacking P.B., Houck J.R., Soifer B.T., Weisstein E.W., 1996, ApJ 456, 428

Aussel H., 1999, Ph.D. Thesis, Univ. Paris VII

Aussel H., Cesarski C.J., Elbaz D., Starck J.L., 1999, A\&A 342, 313

Bertin E., Dennefeld M., Moshir M., 1997, A\&A 323, 685

Clements D.L., Désert F.-X., Franceschini A., et al., A\&A (submitted) astro-ph/9901267

Désert F.X., et al., 1999 (in preparation)

Downes A.J.B., Peacock J.E., Savage A., Carrie D.R., 1986, MNRAS 218, 31

Elbaz D., Aussel H., Cesarski C.J., et al., 1999, in: The Universe as seen by ISO, Cox P. \& Kessler M.F. (eds.). ESA Special Pub. Ser. SP-427 (in press)

Franceschini A., De Zotti G., Toffolatti L., Mazzei P., Danese L., 1991, A\&AS 98, 285

Franceschini A., Mazzei P., De Zotti G., Danese L., 1994, ApJ 427,140

Gregorich D.T., Neugebauer G., Soifer B.T., Gunn J.E., Herter T.L., 1995, AJ 110, 259

Hacking P., 1987, Ph.D. Thesis, Cornell University

Hacking P., Condon J.J., Houck J.R., 1987, ApJ 316, L15

Hacking P., Condon J.J., Houck J.R., Beichman C.A., 1989, ApJ 339, 12

Hacking P., Houck J.R., 1987, ApJS 63, 311

Hogg D.W., Turner E.L., 1998, PASP 110, 727

Leech K., 1998, Infrared Space Observatory (ISO) Satellite \& Data Manual, Issue 1, SAI/98-094/Dc, www.iso.vilspa.esa.es/manuals/isoidum4/isoidum.html

Moshir M.M., et al., 1997, Explanatory Supplement to the IRAS Faint Source Survey, Version 2, JPL D-10015, Pasadena

Okumura K., 1997, ISOCAM PSF Report, ESA/CAM IDT, available at www.estec.esa.nl:80/instr/CAM/calwksp/

Oliver S., Rowan-Robinson M., Cesarsky C., et al., 1998, in: Wide Field Surveys in Cosmology. Ed. Frontières, p. 165

Oliver S.J., Rowan-Robinson M., Saunders W., 1992, MNRAS 256, 15P

Ott S., et al., 1998, Astronomical Data Analysis Software \& Systems, Albrecht R., Hook R.N. \& Bushouse H.A. (eds.), ASP Conf. Ser. 145, 275

Rowan-Robinson M., et al., 1999, in: The Universe as seen by ISO, Cox P. and Kessler M.F. (eds.), ESA SP-427 (in press) astro-ph/9906273

Siebenmorgen R., Blommaert J., Sauvage M., Starck J.-L., 1999, ISO Handbook Volume III (CAM), Version 1.0 SAI99-057/Dc

Starck J.L., Abergel A., Aussel H., et al., 1999, A\&AS 134, 135

Starck J.L., Claret A., Siebenmorgen R., 1996, ISOCAM Data Calibration, CEA Saclay Technical Report

Sutherland W., Saunders W., 1992, ApJ 259, 413

Treyer M.A., Silk J., 1993, ApJ 408, L1

Wolstencroft R.D., Savage A., Clowes R.G., MacGillivray H.T., Leggett S.K., Kalafi M., 1986, MNRAS 223, 279 
Table 1. Observing log

\begin{tabular}{|c|c|c|c|c|c|c|}
\hline $\begin{array}{l}\text { HH87 } \\
\text { name }\end{array}$ & $\begin{array}{c}\text { IRAS FSS } \\
\text { name }\end{array}$ & $\begin{array}{c}\alpha \\
(2000)\end{array}$ & $\begin{array}{c}\delta \\
(2000)\end{array}$ & $\begin{array}{l}\text { Time on } \\
\text { source }(\mathrm{s})\end{array}$ & $\begin{array}{c}\text { Observation } \\
\text { date }\end{array}$ & $\begin{array}{c}\text { TDTOSN } \\
\text { no. }\end{array}$ \\
\hline $3-01$ & $17473+6728$ & $17^{\mathrm{h}} 47^{\mathrm{m}} 15^{\mathrm{s}} \cdot 2$ & $67^{\circ} 27^{\prime} 52^{\prime \prime}$ & 342 & 19 Dec. 96 & 39803702 \\
\hline $3-02$ & $17479+6735$ & 174750.8 & 673453 & 342 & 13 Feb. 97 & 45500302 \\
\hline $3-03$ & $17479+6633$ & 174800.4 & 663224 & 218 & 19 Dec. 96 & 39901504 \\
\hline $3-04$ & $17480+6657$ & 174801.8 & 665629 & 216 & 19 Dec. 96 & 39803706 \\
\hline $3-05$ & $17481+6629$ & $1748 \quad 10.6$ & 662842 & 218 & 19 Dec. 96 & 39901508 \\
\hline $3-06$ & $17482+6638$ & $1748 \quad 14.1$ & 663745 & 218 & 19 Dec. 96 & 39803710 \\
\hline $3-07$ & $17484+6615$ & 174827.0 & $66 \quad 1412$ & 218 & 19 Dec. 96 & 39901512 \\
\hline $3-08$ & $17487+6742$ & 174833.8 & 674157 & 286 & 19 Dec. 96 & 39803714 \\
\hline $3-09$ & $17488+6654$ & 174848.7 & 665343 & 218 & 19 Dec. 96 & 39803716 \\
\hline $3-10$ & $17493+6626$ & 174921.2 & 662551 & 220 & 19 Dec. 96 & 39901519 \\
\hline $3-11$ & $17493+6721$ & 174914.4 & 672024 & 218 & 19 Dec. 96 & 39803721 \\
\hline $3-12$ & $17496+6652$ & 174937.5 & 665123 & 353 & 19 Dec. 96 & 39901523 \\
\hline $3-13$ & $17497+6727$ & 174936.8 & 672702 & 218 & 19 Dec. 96 & 39803725 \\
\hline $3-14$ & $17503+6539$ & 175030.0 & 653824 & 221 & 19 Dec. 96 & 39901528 \\
\hline $3-15$ & $17504+6722$ & 175021.5 & 672119 & 216 & 19 Dec. 96 & 39803731 \\
\hline $3-16$ & $17505+6700$ & 175027.8 & 665957 & 218 & 19 Dec. 96 & 39901533 \\
\hline $3-17$ & $17505+6648$ & 175033.5 & $6648 \quad 17$ & 218 & 19 Dec. 96 & 39901536 \\
\hline $3-18$ & $17512+6747$ & 175106.2 & 674632 & 216 & 19 Dec. 96 & 39803739 \\
\hline $3-19$ & $17514+6534$ & 175134.7 & 653407 & 218 & 19 Dec. 96 & 39901541 \\
\hline $3-20$ & $17514+6713$ & 175123.2 & 671231 & 235 & 19 Dec. 96 & 39803743 \\
\hline $3-21$ & $17523+6626$ & 175222.6 & 662608 & 242 & 19 Dec. 96 & 39901545 \\
\hline $3-22$ & $17526+6739$ & 175228.2 & 673915 & 361 & 19 Dec. 96 & 39901647 \\
\hline $3-23$ & $17527+6530$ & 175252.4 & 653018 & 349 & 19 Dec. 96 & 39901749 \\
\hline $3-24$ & $17538+6639$ & 175349.0 & 663930 & 216 & 19 Dec. 96 & 39901651 \\
\hline $3-25$ & $17540+6544$ & 175409.4 & 654432 & 214 & 19 Dec. 96 & 39901754 \\
\hline $3-26$ & $17546+6623$ & 175440.2 & 662304 & 214 & 19 Dec. 96 & 39901656 \\
\hline $3-27$ & $17547+6648$ & 175442.1 & 664832 & 214 & 19 Dec. 96 & 39901658 \\
\hline $3-28$ & $17548+6554$ & 175455.8 & 655349 & 216 & 19 Dec. 96 & 39901760 \\
\hline $3-29$ & $17548+6613$ & 175454.6 & $66 \quad 1248$ & 214 & 19 Dec. 96 & 39901763 \\
\hline $3-30$ & $17550+6710$ & 175457.1 & 671007 & 216 & 19 Dec. 96 & 39901666 \\
\hline $3-31$ & $17552+6632$ & 175517.0 & 663224 & 214 & 19 Dec. 96 & 39901769 \\
\hline $3-32$ & $17553+6557$ & 175526.9 & 655655 & 214 & 19 Dec. 96 & 39901771 \\
\hline $3-33$ & $17553+6624$ & 175524.1 & 662407 & 216 & 19 Dec. 96 & 39901674 \\
\hline $3-34$ & $17557+6540$ & 175550.3 & 653946 & 214 & 19 Dec. 96 & 39901777 \\
\hline $3-35$ & $17559+6610$ & 175556.6 & 660942 & 214 & 19 Dec. 96 & 39901780 \\
\hline $3-36$ & $17559+6748$ & 175546.2 & 674760 & 214 & 19 Dec. 96 & 39901682 \\
\hline $3-37$ & $17560+6720$ & 175554.9 & 672008 & 214 & 19 Dec. 96 & 39901685 \\
\hline $3-38$ & $17562+6647$ & 175611.2 & 664652 & 231 & 19 Dec. 96 & 39901787 \\
\hline $3-39$ & $17562+6744$ & 175606.0 & 674403 & 298 & 19 Dec. 96 & 39901690 \\
\hline $3-40$ & $17566+6724$ & 175632.0 & 672404 & 342 & 13 Feb. 97 & 45500424 \\
\hline $3-41$ & $17566+6648$ & 175638.6 & $\begin{array}{lll}66 & 48 & 17\end{array}$ & 403 & 13 Feb. 97 & 45500525 \\
\hline $3-42$ & $17568+6546$ & 175657.7 & 654551 & 338 & 20 Dec. 96 & 40000505 \\
\hline $3-43$ & $17570+6730$ & 175655.3 & 672947 & 338 & 20 Dec. 96 & 40000407 \\
\hline $3-44$ & $17575+6719$ & 175728.4 & 671944 & 216 & 20 Dec. 96 & 40000409 \\
\hline $3-45$ & $17576+6745$ & 175731.2 & 674548 & 216 & 20 Dec. 96 & 40000411 \\
\hline $3-46$ & $17576+6704$ & 175736.9 & 670416 & 216 & 20 Dec. 96 & 40000413 \\
\hline $3-47$ & $17581+6529$ & 175813.3 & 652859 & 214 & 20 Dec. 96 & 40000516 \\
\hline $3-48$ & $17583+6539$ & 175814.1 & 653848 & 216 & 20 Dec. 96 & 40000518 \\
\hline $3-49$ & $17582+6552$ & 175816.6 & 655218 & 214 & 20 Dec. 96 & 40000520 \\
\hline $3-50$ & $17583+6629$ & 175820.4 & 662856 & 216 & 20 Dec. 96 & 40000422 \\
\hline $3-51$ & $17583+6539$ & 175838.3 & 654020 & 216 & 20 Dec. 96 & 40000524 \\
\hline $3-53$ & $17586+6744$ & 175830.6 & 674404 & 214 & 20 Dec. 96 & 40000427 \\
\hline $3-54$ & $17587+6607$ & 175848.4 & 660721 & 216 & 20 Dec. 96 & 40000529 \\
\hline $3-55$ & $17588+6648$ & 175847.8 & $66 \quad 48 \quad 13$ & 216 & 20 Dec. 96 & 40000432 \\
\hline
\end{tabular}


Table 1. continued

\begin{tabular}{|c|c|c|c|c|c|c|}
\hline $\begin{array}{l}\text { HH87 } \\
\text { name }\end{array}$ & $\begin{array}{c}\begin{array}{c}\text { IRAS FSS } \\
\text { name }\end{array} \\
\text { ame }\end{array}$ & $\begin{array}{c}\alpha \\
(2000)\end{array}$ & $\begin{array}{c}\delta \\
(2000)\end{array}$ & $\begin{array}{l}\text { Time on } \\
\text { source }(\mathrm{s})\end{array}$ & $\begin{array}{c}\text { Observation } \\
\text { date }\end{array}$ & $\begin{array}{c}\text { TDTOSN } \\
\text { no. }\end{array}$ \\
\hline $3-56$ & $17589+6716$ & 175848.1 & 671601 & 214 & 20 Dec. 96 & 40000434 \\
\hline $3-57$ & $17590+6616$ & 175906.8 & 661608 & 216 & 20 Dec. 96 & 40000536 \\
\hline $3-58$ & $17591+6625$ & 175908.7 & 662457 & 216 & 20 Dec. 96 & 40000538 \\
\hline $3-59$ & $17592+6606$ & 175918.3 & 660626 & 214 & 20 Dec. 96 & 40000540 \\
\hline $3-61$ & $18002+6727$ & 180009.0 & 672736 & 216 & 20 Dec. 96 & 40000442 \\
\hline $3-62$ & $18003+6529$ & 180028.4 & 652908 & 231 & 20 Dec. 96 & 40000545 \\
\hline $3-63$ & $18010+6721$ & 180056.7 & 672129 & 235 & 20 Dec. 96 & 40000447 \\
\hline $3-64$ & $18012+6725$ & 180106.7 & 672533 & 345 & 20 Dec. 96 & 40000650 \\
\hline $3-65$ & $18015+6700$ & 180128.5 & 670036 & 216 & 20 Dec. 96 & 40000652 \\
\hline $3-66$ & $18018+6658$ & 180148.6 & 665903 & 216 & 20 Dec. 96 & 40000655 \\
\hline $3-67$ & $18019+6553$ & 180159.8 & 655338 & 330 & 20 Dec. 96 & 40000758 \\
\hline $3-68$ & $18019+6708$ & 180151.0 & 670848 & 214 & 20 Dec. 96 & 40000660 \\
\hline $3-69$ & $18028+6659$ & 180246.2 & $6700 \quad 02$ & 216 & 20 Dec. 96 & 40000662 \\
\hline $3-70$ & $18030+6623$ & 180301.1 & 662356 & 214 & 20 Dec. 96 & 40000765 \\
\hline $3-71$ & $18033+6650$ & 180320.6 & 665036 & 214 & 20 Dec. 96 & 40000768 \\
\hline $3-72$ & $18034+6732$ & 180320.0 & 673243 & 214 & 20 Dec. 96 & 40000671 \\
\hline $3-73$ & $18037+6702$ & 180343.6 & 670255 & 214 & 20 Dec. 96 & 40000673 \\
\hline $3-74$ & $18040+6635$ & 180359.3 & 663522 & 216 & 20 Dec. 96 & 40000775 \\
\hline $3-75$ & $18043+6729$ & 180411.0 & 672932 & 214 & 20 Dec. 96 & 40000678 \\
\hline $3-76$ & $18044+6720$ & 180423.7 & 672047 & 216 & 20 Dec. 96 & 40000681 \\
\hline $3-77$ & $18046+6549$ & 180445.5 & 654924 & 214 & 20 Dec. 96 & 40000784 \\
\hline $3-79$ & $18052+6653$ & 180515.1 & 665348 & 216 & 20 Dec. 96 & 40000687 \\
\hline $3-78$ & $18052+6707$ & $\begin{array}{lll}18 & 05 & 07.2\end{array}$ & 670744 & 231 & 20 Dec. 96 & 40000790 \\
\hline $3-80$ & $18053+6711$ & 180516.5 & 671210 & 216 & 20 Dec. 96 & 40000792 \\
\hline $3-81$ & $18054+6554$ & 180531.8 & 655434 & 214 & 20 Dec. 96 & 40000794 \\
\hline $3-82$ & $18055+6644$ & 180529.2 & 664446 & 216 & 20 Dec. 96 & 40000796 \\
\hline $3-83$ & $18064+6620$ & 180627.4 & 662031 & 237 & 20 Dec. 96 & 40000797 \\
\hline $3-84$ & $18066+6632$ & 180638.6 & 663236 & 355 & 20 Dec. 96 & 40000805 \\
\hline $3-85$ & $18073+6701$ & $1807 \quad 19.3$ & 670219 & 214 & 20 Dec. 96 & 40000808 \\
\hline $3-86$ & $18074+6533$ & 180735.7 & 653355 & 342 & 19 Dec. 96 & 39901810 \\
\hline $3-87$ & $18077+6643$ & 180743.2 & 664336 & 214 & 20 Dec. 96 & 40000813 \\
\hline $3-88$ & $18091+6611$ & 180910.3 & $66 \quad 12 \quad 24$ & 218 & 19 Dec. 96 & 39901816 \\
\hline $3-89$ & $18096+6734$ & 180927.9 & 673450 & 216 & 20 Dec. 96 & 40000819 \\
\hline $3-90$ & $18096+6649$ & 180937.2 & 665006 & 214 & 20 Dec. 96 & 40000821 \\
\hline $3-91$ & $18099+6707$ & 180952.3 & 670835 & 216 & 20 Dec. 96 & 40000823 \\
\hline $3-92$ & $18104+6641$ & 181025.5 & 664158 & 296 & 19 Dec. 96 & 39901826 \\
\hline $3-93$ & $18105+6610$ & 181033.7 & 661136 & 300 & 19 Dec. 96 & 39901829 \\
\hline $3-94$ & $18108+6532$ & 181100.4 & 653319 & 326 & 19 Dec. 96 & 39901832 \\
\hline $3-95$ & $18108+6715$ & 181048.0 & 671634 & 298 & 20 Dec. 96 & 40000835 \\
\hline $3-96$ & $18110+6744$ & 181054.8 & 674511 & 504 & 19 Dec. 96 & 39901838 \\
\hline $2-16$ & $18002+6652$ & 180011.2 & 665216 & 342 & 13 Feb. 97 & 45500636 \\
\hline
\end{tabular}


Table 2. ISOCAM sources

\begin{tabular}{|c|c|c|c|c|c|c|}
\hline Target & $\alpha(2000)$ & $\overline{\delta(2000)}$ & 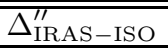 & $S_{14.3 \mu \mathrm{m}}$ & $\overline{S_{\mathrm{ML}}}$ & $\overline{n_{\mathrm{c}}{ }^{1}}$ \\
\hline $3-01$ & $17^{\mathrm{h}} 47^{\mathrm{m}} 24^{\mathrm{s}} .74$ & $67^{\circ} 26^{\prime} 48^{\prime \prime} .3$ & 81.5 & $16.1 \pm 0.8$ & $16.0 \pm 0.8$ & $6.8 \mathrm{E}-3$ \\
\hline $3-02$ & 174803.47 & 673522.8 & 104.8 & $6.9 \pm 0.9$ & $6.6 \pm 0.9$ & $2.7 \mathrm{E}-2$ \\
\hline $3-03$ & 174800.51 & $\begin{array}{llll}66 & 32 & 17.9\end{array}$ & 6.6 & $8.8 \pm 0.9$ & $8.6 \pm 0.9$ & $7.5 \mathrm{E}-5$ \\
\hline $3-04$ & 174804.57 & $66 \quad 5623.5$ & 7.1 & $12.1 \pm 1.0$ & $11.9 \pm 1.0$ & $9.5 \mathrm{E}-6$ \\
\hline $3-05$ & 174808.77 & 662841.8 & 11.3 & $8.2 \pm 1.0$ & $7.9 \pm 1.0$ & $3.3 \mathrm{E}-4$ \\
\hline $3-06$ & & & & $<1.5$ & & \\
\hline $3-07$ & 174827.11 & $66 \quad 1405.9$ & 7.4 & $3.1 \pm 0.7$ & & $3.9 \mathrm{E}-4$ \\
\hline $3-08$ & 174837.75 & $\begin{array}{llll}67 & 41 & 57.3\end{array}$ & 1.6 & $13.8 \pm 0.8$ & $13.7 \pm 0.8$ & $3.6 \mathrm{E}-5$ \\
\hline $3-09$ & 174850.44 & $6653 \quad 55.3$ & 17.6 & $7.0 \pm 0.8$ & $6.8 \pm 0.8$ & $6.5 \mathrm{E}-4$ \\
\hline $3-10$ & 174922.31 & 662556.5 & 8.3 & $16.9 \pm 0.9$ & $16.8 \pm 0.9$ & $5.7 \mathrm{E}-5$ \\
\hline $3-11$ & 174915.21 & 672012.8 & 7.4 & $8.5 \pm 1.0$ & $8.2 \pm 1.0$ & $3.5 \mathrm{E}-4$ \\
\hline $3-12$ & 174933.72 & 665122.9 & 23.4 & $11.6 \pm 0.9$ & $11.4 \pm 0.9$ & $8.5 \mathrm{E}-4$ \\
\hline $3-13$ & 174932.55 & 672656.9 & 29.2 & $2.0 \pm 0.4$ & $1.8 \pm 0.4$ & $1.1 \mathrm{E}-2$ \\
\hline $3-14$ & 175030.97 & $\begin{array}{lll}65 & 38 & 11.9\end{array}$ & 13.5 & $3.1 \pm 0.9$ & & $1.4 \mathrm{E}-3$ \\
\hline $3-15$ & 175021.23 & 672102.1 & 14.3 & $9.7 \pm 0.9$ & $9.5 \pm 0.9$ & $5.9 \mathrm{E}-4$ \\
\hline $3-16$ & 175025.01 & $6700 \quad 08.7$ & 20.9 & $3.1 \pm 0.8$ & & $4.1 \mathrm{E}-3$ \\
\hline \multirow[t]{2}{*}{$3-17$} & 175026.69 & 664822.7 & 42.1 & $3.1 \pm 0.7$ & & $1.6 \mathrm{E}-2$ \\
\hline & 175034.62 & 664828.4 & 12.8 & $3.6 \pm 0.9$ & & $1.2 \mathrm{E}-3$ \\
\hline $3-18$ & 175104.04 & 674720.1 & 56.3 & $1.5 \pm 0.4$ & & $6.1 \mathrm{E}-2$ \\
\hline \multirow[t]{3}{*}{$3-19$} & 175137.58 & 653348.9 & 26.2 & $4.2 \pm 0.9$ & & $3.6 \mathrm{E}-3$ \\
\hline & 175131.98 & 653343.4 & 30.6 & $2.4 \pm 0.6$ & & $1.1 \mathrm{E}-2$ \\
\hline & 175130.04 & 653412.8 & 30.6 & $2.0 \pm 0.7$ & & $1.5 \mathrm{E}-2$ \\
\hline \multirow[t]{2}{*}{$3-20$} & 175117.97 & $67 \quad 1231.7$ & 34.6 & $2.9 \pm 0.7^{2}$ & & $2.5 \mathrm{E}-2$ \\
\hline & 175115.95 & $\begin{array}{lll}67 & 12 & 02.4\end{array}$ & 52.3 & $2.4 \pm 0.6^{3}$ & & $1.2 \mathrm{E}-2$ \\
\hline $3-21$ & 175220.76 & 662556.1 & 17.8 & $4.0 \pm 1.0$ & & $1.9 \mathrm{E}-3$ \\
\hline $3-22$ & & & & $<1.7$ & & \\
\hline $3-23$ & 175253.37 & 653005.9 & 15.0 & $3.4 \pm 0.7$ & & $1.5 \mathrm{E}-3$ \\
\hline $3-24$ & 175348.14 & 663923.9 & 9.1 & $7.1 \pm 0.9$ & $6.8 \pm 0.9$ & $2.3 \mathrm{E}-4$ \\
\hline $3-25$ & 175411.41 & 654449.2 & 20.7 & $8.3 \pm 0.9$ & $8.1 \pm 0.9$ & $1.0 \mathrm{E}-3$ \\
\hline \multirow[t]{4}{*}{$3-26$} & 175438.39 & 662321.5 & 21.3 & $4.4 \pm 0.7$ & $4.1 \pm 0.7$ & $2.7 \mathrm{E}-3$ \\
\hline & 175437.38 & 662246.3 & 26.3 & $8.2 \pm 0.9$ & $8.0 \pm 0.9$ & $1.6 \mathrm{E}-3$ \\
\hline & 175447.20 & $\begin{array}{lll}66 & 23 & 19.4\end{array}$ & 44.8 & $5.9 \pm 0.9$ & $5.6 \pm 0.9$ & $7.1 \mathrm{E}-3$ \\
\hline & 175445.44 & $6623 \quad 50.2$ & 55.7 & $1.5 \pm 0.7$ & & $7.9 \mathrm{E}-2$ \\
\hline \multirow[t]{2}{*}{$3-27$} & 175446.19 & 664825.5 & 26.1 & $4.7 \pm 0.6$ & $4.5 \pm 0.6$ & $3.1 \mathrm{E}-3$ \\
\hline & 175439.44 & 664925.1 & 55.6 & $8.1 \pm 1.0$ & $7.8 \pm 1.0$ & $7.7 \mathrm{E}-3$ \\
\hline $3-28$ & 175457.91 & 655406.2 & 21.4 & $6.1 \pm 0.9$ & $5.8 \pm 0.9$ & $1.6 \mathrm{E}-3$ \\
\hline $3-29$ & 175455.75 & $6613 \quad 11.2$ & 24.3 & $3.9 \pm 1.0$ & & $3.9 \mathrm{E}-3$ \\
\hline $3-30$ & 175451.18 & 670950.2 & 41.0 & $3.0 \pm 1.0$ & & $1.5 \mathrm{E}-2$ \\
\hline $3-31$ & 175518.10 & 663223.6 & 5.8 & $18.1 \pm 1.5$ & $17.8 \pm 1.5$ & $2.0 \mathrm{E}-5$ \\
\hline \multirow[t]{2}{*}{$3-32$} & 175533.77 & 655704.5 & 43.6 & $6.4 \pm 1.0$ & $6.0 \pm 1.0$ & $6.0 \mathrm{E}-3$ \\
\hline & 175511.67 & 655654.5 & 95.8 & $2.1 \pm 0.8$ & & $1.4 \mathrm{E}-1$ \\
\hline \multirow[t]{2}{*}{$3-33$} & 175521.31 & 662424.6 & 24.0 & $5.0 \pm 0.9$ & $4.6 \pm 0.8$ & $2.8 \mathrm{E}-3$ \\
\hline & 175520.12 & 662303.4 & 72.3 & $3.1 \pm 0.7$ & & $4.3 \mathrm{E}-2$ \\
\hline $3-34$ & 175551.28 & 653939.8 & 9.0 & $4.6 \pm 0.8$ & $4.3 \pm 0.7$ & $3.1 \mathrm{E}-4$ \\
\hline $3-35$ & 175559.64 & $\begin{array}{lll}66 & 09 & 41.4\end{array}$ & 31.5 & $2.3 \pm 1.0$ & & $1.3 \mathrm{E}-2$ \\
\hline \multirow[t]{2}{*}{$3-36$} & 175545.34 & 674753.9 & 8.2 & $5.6 \pm 0.9$ & $5.2 \pm 0.8$ & $2.4 \mathrm{E}-4$ \\
\hline & 175549.09 & 674656.2 & 69.9 & $5.5 \pm 0.8$ & $5.2 \pm 0.8$ & $1.8 \mathrm{E}-2$ \\
\hline $3-37$ & 175555.12 & $\begin{array}{lll}67 & 20 & 07.7\end{array}$ & 2.0 & $21.9 \pm 0.9$ & $21.8 \pm 0.9$ & $6.4 \mathrm{E}-7$ \\
\hline \multirow[t]{2}{*}{$3-38$} & 175606.55 & 664739.2 & 56.2 & $4.0 \pm 1.0$ & & $2.0 \mathrm{E}-2$ \\
\hline & 175615.31 & 664650.3 & 23.9 & $2.6 \pm 0.7$ & & $5.8 \mathrm{E}-3$ \\
\hline $3-39$ & 175607.17 & $6743 \quad 50.9$ & 14.2 & $5.1 \pm 0.8$ & $4.8 \pm 0.8$ & $7.5 \mathrm{E}-4$ \\
\hline \multirow[t]{2}{*}{$3-40$} & 175626.95 & 672358.3 & 27.7 & $7.8 \pm 0.9$ & $7.6 \pm 0.9$ & $2.6 \mathrm{E}-3$ \\
\hline & 175636.41 & 672423.9 & 38.6 & $1.8 \pm 0.7^{4}$ & & $1.8 \mathrm{E}-2$ \\
\hline $3-41$ & 175639.72 & $6648 \quad 00.3$ & 15.2 & $9.7 \pm 1.0$ & $9.5 \pm 1.0$ & $6.5 \mathrm{E}-4$ \\
\hline $3-42$ & 175652.25 & 654608.5 & 39.1 & $4.5 \pm 0.7$ & $4.2 \pm 0.7$ & $8.4 \mathrm{E}-3$ \\
\hline $3-43$ & 175657.78 & 672952.4 & 18.6 & $10.2 \pm 1.0$ & $10.0 \pm 1.0$ & $3.4 \mathrm{E}-4$ \\
\hline $3-44$ & 175731.89 & 672001.1 & 24.4 & $3.4 \pm 0.9$ & & $5.3 \mathrm{E}-3$ \\
\hline
\end{tabular}


Table 2. continued

\begin{tabular}{|c|c|c|c|c|c|c|}
\hline Target & $\alpha(2000)$ & $\delta(2000)$ & $\Delta_{\text {IRAS-ISO }}^{\prime \prime}$ & $S_{14.3 \mu \mathrm{m}}$ & $S_{\mathrm{ML}}$ & $n_{\mathrm{c}}$ \\
\hline $3-45$ & 175732.61 & 674553.5 & 6.7 & $9.5 \pm 1.0$ & $9.3 \pm 1.0$ & $1.3 \mathrm{E}-4$ \\
\hline $3-46$ & 175737.22 & 670415.7 & 2.9 & $1.4 \pm 0.7$ & & $6.2 \mathrm{E}-7$ \\
\hline $3-47$ & 175811.64 & 652835.4 & 28.9 & $8.8 \pm 0.9$ & $8.6 \pm 0.9$ & $1.5 \mathrm{E}-3$ \\
\hline $3-48$ & $1758 \quad 17.29$ & 653905.1 & 23.6 & $13.4 \pm 0.9$ & $13.3 \pm 0.9$ & $8.0 \mathrm{E}-4$ \\
\hline $3-49$ & 175816.95 & 655235.4 & 14.6 & $2.9 \pm 0.7$ & & $2.8 \mathrm{E}-3$ \\
\hline $3-50$ & 175821.71 & 662901.5 & 6.7 & $6.7 \pm 1.0$ & $6.3 \pm 1.0$ & $2.2 \mathrm{E}-4$ \\
\hline \multirow[t]{2}{*}{$3-51$} & 175838.64 & 654031.5 & 9.0 & $3.5 \pm 0.7$ & $3.1 \pm 0.6$ & $1.0 \mathrm{E}-3$ \\
\hline & 175841.85 & 653854.0 & 96.2 & $2.1 \pm 0.5$ & & $1.18 \mathrm{E}-1$ \\
\hline $3-53$ & 175831.03 & 674409.6 & 3.2 & $2.1 \pm 0.9$ & & $5.2 \mathrm{E}-4$ \\
\hline $3-54$ & 175850.55 & $\begin{array}{lll}66 & 07 & 14.7\end{array}$ & 14.6 & $1.5 \pm 0.7$ & & $3.9 \mathrm{E}-3$ \\
\hline $3-55$ & 175847.13 & 664801.0 & 15.6 & $2.6 \pm 0.8$ & & $1.8 \mathrm{E}-3$ \\
\hline $3-56$ & 175847.44 & 671554.9 & 10.7 & $10.6 \pm 1.0$ & $10.4 \pm 1.0$ & $1.2 \mathrm{E}-4$ \\
\hline $3-57$ & 175905.17 & $\begin{array}{lll}66 & 16 & 07.8\end{array}$ & 11.3 & $8.7 \pm 0.9$ & $8.5 \pm 0.9$ & $2.5 \mathrm{E}-4$ \\
\hline $3-58$ & 175909.05 & $\begin{array}{lll}66 & 2514.4\end{array}$ & 14.7 & $4.6 \pm 0.7$ & $4.3 \pm 0.7$ & $1.6 \mathrm{E}-3$ \\
\hline $3-59$ & 175918.64 & 660637.5 & 9.1 & $5.3 \pm 1.0$ & $4.8 \pm 0.9$ & $6.0 \mathrm{E}-4$ \\
\hline $3-61$ & 180008.36 & 672741.7 & 6.0 & $9.2 \pm 0.9$ & $9.0 \pm 0.9$ & $1.2 \mathrm{E}-4$ \\
\hline $3-62$ & 180026.77 & 652913.7 & 12.0 & $6.0 \pm 0.6$ & $5.9 \pm 0.6$ & $5.7 \mathrm{E}-4$ \\
\hline \multirow[t]{2}{*}{$3-63$} & 180056.06 & 672134.7 & 6.3 & $13.5 \pm 1.0$ & $13.3 \pm 1.0$ & $7.1 \mathrm{E}-5$ \\
\hline & 180043.92 & 672026.9 & 104.3 & $8.9 \pm 0.9$ & $8.7 \pm 0.9$ & $2.1 \mathrm{E}-2$ \\
\hline $3-64$ & $\begin{array}{lll}18 & 01.02\end{array}$ & $\begin{array}{lll}67 & 2532.7\end{array}$ & 3.6 & $25.0 \pm 1.3$ & $24.8 \pm 1.3$ & $1.0 \mathrm{E}-6$ \\
\hline $3-65$ & 180126.89 & 670053.5 & 18.7 & $3.5 \pm 0.9$ & & $3.2 \mathrm{E}-3$ \\
\hline $3-66$ & 180150.02 & 665914.4 & 10.7 & $2.4 \pm 0.7$ & & $2.2 \mathrm{E}-3$ \\
\hline \multirow[t]{2}{*}{$3-67$} & 180149.67 & 655425.6 & 78.4 & $5.5 \pm 0.8$ & $5.2 \pm 0.8$ & $2.6 \mathrm{E}-2$ \\
\hline & 180149.67 & 655425.6 & 78.4 & $5.5 \pm 0.8$ & $5.2 \pm 0.8$ & $2.6 \mathrm{E}-2$ \\
\hline $3-68$ & 180145.36 & 670900.6 & 35.6 & $3.8 \pm 0.9$ & & $8.7 \mathrm{E}-3$ \\
\hline $3-69$ & 180248.59 & $\begin{array}{lll}67 & 00 & 12.3\end{array}$ & 15.2 & $2.6 \pm 0.8$ & & $3.0 \mathrm{E}-3$ \\
\hline $3-70$ & 180301.41 & 662349.8 & 8.4 & $6.1 \pm 0.8$ & $5.8 \pm 0.8$ & $1.1 \mathrm{E}-4$ \\
\hline $3-71$ & 180321.03 & 665041.6 & 3.1 & $4.8 \pm 0.9$ & $4.4 \pm 0.8$ & $1.7 \mathrm{E}-4$ \\
\hline $3-72$ & 180324.77 & 673138.1 & 75.4 & $4.1 \pm 0.7$ & $3.8 \pm 0.7$ & $3.0 \mathrm{E}-2$ \\
\hline $3-73$ & 180341.99 & 670306.6 & 13.6 & $18.9 \pm 0.9$ & $18.8 \pm 0.9$ & $1.8 \mathrm{E}-4$ \\
\hline $3-74$ & 180400.61 & 663527.5 & 14.9 & $7.2 \pm 0.9$ & $6.9 \pm 0.9$ & $9.3 \mathrm{E}-4$ \\
\hline $3-75$ & 180407.32 & 672932.8 & 23.6 & $1.4 \pm 0.5$ & & $1.4 \mathrm{E}-2$ \\
\hline $3-76$ & 180427.13 & 672045.4 & 20.7 & $10.2 \pm 1.0$ & $10.0 \pm 1.0$ & $7.3 \mathrm{E}-4$ \\
\hline $3-77$ & 180452.78 & 655001.9 & 57.1 & $4.2 \pm 0.9$ & & $2.0 \mathrm{E}-2$ \\
\hline \multirow[t]{3}{*}{$3-78$} & 180508.13 & 670854.6 & 68.1 & $3.9 \pm 0.9$ & & $3.2 \mathrm{E}-2$ \\
\hline & 180509.56 & 670742.6 & 13.1 & $2.8 \pm 0.6$ & & $1.5 \mathrm{E}-3$ \\
\hline & 180508.32 & $6707 \quad 13.5$ & 34.6 & $1.5 \pm 0.6$ & & $2.4 \mathrm{E}-2$ \\
\hline \multirow[t]{3}{*}{$3-79$} & 180517.61 & 665404.2 & 19.6 & $15.1 \pm 0.9$ & $15.0 \pm 0.9$ & $4.8 \mathrm{E}-4$ \\
\hline & 180525.03 & 665448.6 & 83.2 & $31.9 \pm 1.0$ & $31.8 \pm 1.0$ & $2.8 \mathrm{E}-3$ \\
\hline & 180459.66 & 665448.2 & 110.7 & $6.3 \pm 0.7^{5}$ & $6.1 \pm 0.7$ & $4.2 \mathrm{E}-2$ \\
\hline \multirow[t]{2}{*}{$3-80$} & 180514.89 & 671221.6 & 13.7 & $23.4 \pm 1.0$ & $23.3 \pm 1.0$ & $1.4 \mathrm{E}-4$ \\
\hline & 180516.81 & 671203.8 & 12.6 & $22.2 \pm 1.1$ & $22.1 \pm 1.1$ & $2.8 \mathrm{E}-5$ \\
\hline \multirow[t]{2}{*}{$3-81$} & 180528.22 & 655434.8 & 23.4 & $5.9 \pm 0.7$ & $5.7 \pm 0.7$ & $1.9 \mathrm{E}-3$ \\
\hline & 180536.59 & 655408.9 & 41.4 & $2.9 \pm 0.8$ & & $1.4 \mathrm{E}-2$ \\
\hline $3-82$ & 180531.58 & 664450.4 & 6.1 & $12.3 \pm 0.9$ & $12.1 \pm 0.9$ & $7.7 \mathrm{E}-5$ \\
\hline $3-83$ & 180627.60 & 662019.0 & 15.7 & $4.6 \pm 0.9$ & $4.1 \pm 0.8$ & $8.0 \mathrm{E}-4$ \\
\hline $3-84$ & 180639.90 & 663240.5 & 5.8 & $6.7 \pm 0.8$ & $6.5 \pm 0.8$ & $1.5 \mathrm{E}-4$ \\
\hline $3-85$ & 180717.56 & $\begin{array}{lll}67 & 02 & 07.1\end{array}$ & 19.3 & $4.9 \pm 1.0$ & & $1.4 \mathrm{E}-3$ \\
\hline $3-86$ & 180739.78 & 653415.8 & 31.2 & $2.7 \pm 0.8$ & & $1.1 \mathrm{E}-2$ \\
\hline $3-87$ & & & & $<1.5$ & & \\
\hline \multirow[t]{2}{*}{$3-88$} & 180912.73 & 661252.0 & 29.0 & $3.4 \pm 0.8$ & & $7.5 \mathrm{E}-3$ \\
\hline & 180923.53 & 661256.1 & 86.9 & $2.9 \pm 0.7$ & & $7.0 \mathrm{E}-2$ \\
\hline $3-89$ & 180921.81 & 673411.0 & 56.5 & $2.7 \pm 0.8^{6}$ & & $3.0 \mathrm{E}-2$ \\
\hline $3-90$ & 180937.41 & 664959.8 & 8.6 & $6.2 \pm 1.0$ & $5.8 \pm 0.9$ & $1.1 \mathrm{E}-4$ \\
\hline $3-91$ & 180951.87 & $6708 \quad 52.4$ & 15.2 & $3.9 \pm 0.7$ & $3.6 \pm 0.6$ & $2.0 \mathrm{E}-3$ \\
\hline
\end{tabular}


Table 2. continued

\begin{tabular}{cccrrrc}
\hline \hline Target & $\alpha(2000)$ & $\delta(2000)$ & $\Delta_{\text {IRAS }- \text { ISO }}^{\prime \prime}$ & \multicolumn{1}{c}{$S_{14.3 \mu \mathrm{m}}$} & \multicolumn{1}{c}{$S_{\mathrm{ML}}$} & $n_{\mathrm{c}}$ \\
\hline $3-92$ & 181024.42 & 664256.9 & 56.9 & $3.4 \pm 0.7$ & & $2.7 \mathrm{E}-2$ \\
& 181018.54 & 664311.6 & 82.3 & $3.7 \pm 0.6$ & $3.5 \pm 0.6$ & $5.0 \mathrm{E}-2$ \\
& 181024.87 & 664215.4 & 14.6 & $1.8 \pm 0.7$ & & $5.6 \mathrm{E}-3$ \\
$3-93$ & 181034.46 & 661117.1 & 23.0 & $3.3 \pm 0.9$ & & $3.2 \mathrm{E}-3$ \\
$3-94$ & 181100.42 & 653317.7 & 3.7 & $15.4 \pm 1.0$ & $15.3 \pm 1.0$ & $1.8 \mathrm{E}-6$ \\
& 181112.98 & 653246.6 & 89.3 & $3.7 \pm 0.6$ & $3.5 \pm 0.6$ & $5.0 \mathrm{E}-2$ \\
$3-95$ & & & & $<1.6$ & & \\
$3-96$ & 181054.05 & 674516.6 & 5.8 & $14.9 \pm 1.0$ & $14.7 \pm 1.0$ & $7.2 \mathrm{E}-5$ \\
$2-16$ & 180011.85 & 665215.9 & 9.5 & $11.9 \pm 0.9$ & $11.7 \pm 0.9$ & $2.2 \mathrm{E}-5$ \\
\hline \hline
\end{tabular}

Notes to Table 2:

1 The notation $A \mathrm{E}-b$ stands for $A \times 10^{-b}$.

2 The source is reliably detected. Its flux, however, is highly uncertain due to a cosmic ray impact near the source.

3 The image of this source extends over the dead Col. 23; therefore part of its flux is lost. The given value includes a correction for the missing flux, based on the flux distribution in neighboring pixels.

4 Although the flux is measured at a $<3 \sigma$ level, the signal is detected at $>3 \sigma$ in the brightest pixel.

5 Photometry based on the negative imprint produced by beam-switching; the positive one is at the edge of the field.

${ }^{6}$ Photometry based on the negative imprint; the positive one gives a less significant signal $(1.7 \pm 0.7 \mathrm{mJy})$. 

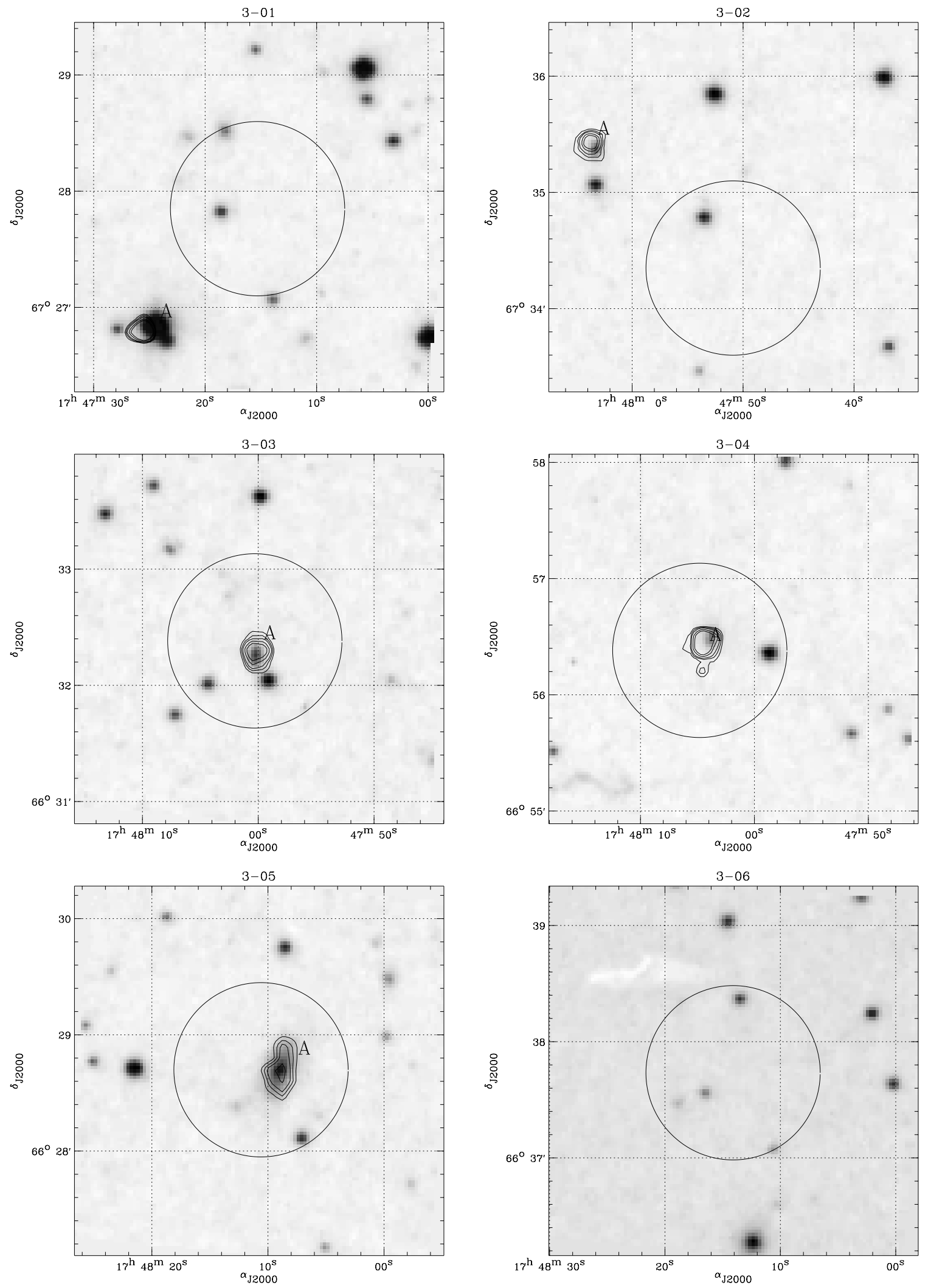

Fig. 4. Finding charts of ISOCAM sources. The large circle is the $45^{\prime \prime}$ IRAS error box. Contours are ISOCAM $15 \mu \mathrm{m}$ detections 
H. Aussel et al.: ISOCAM observations of the IRAS NEPR $60 \mu \mathrm{m}$ sample. I.
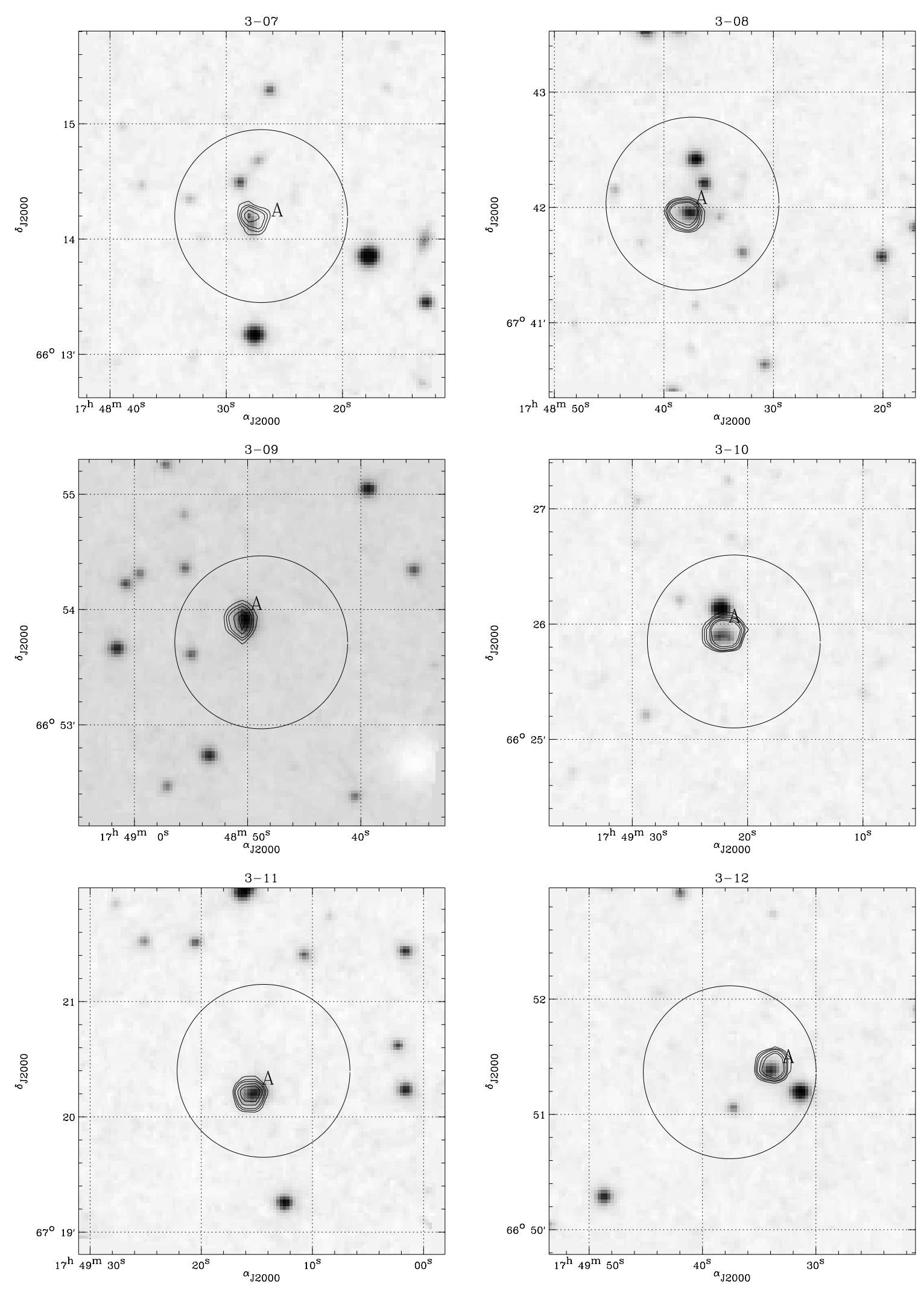

Fig. 4. continued 

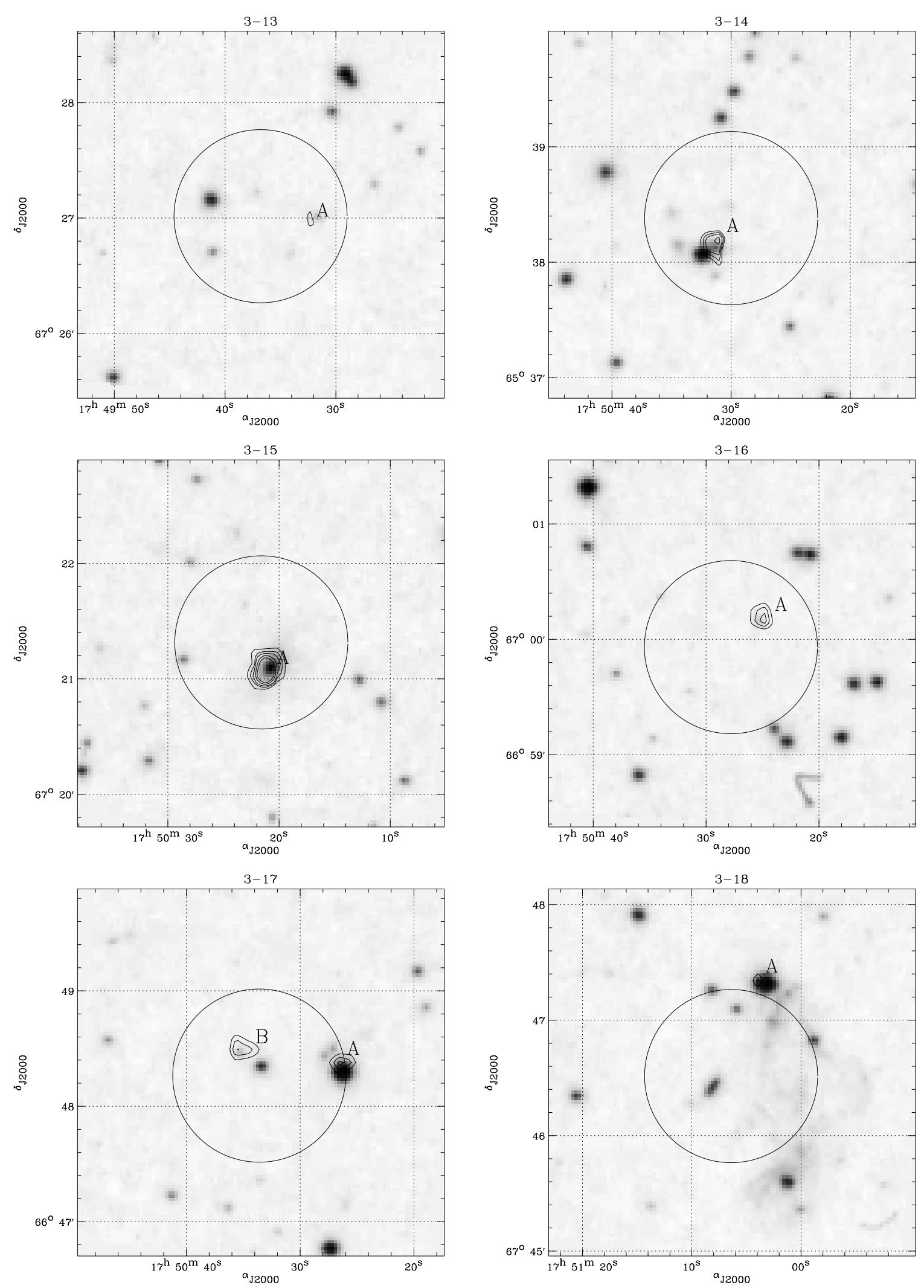

Fig. 4. continued 
H. Aussel et al.: ISOCAM observations of the IRAS NEPR $60 \mu \mathrm{m}$ sample. I.
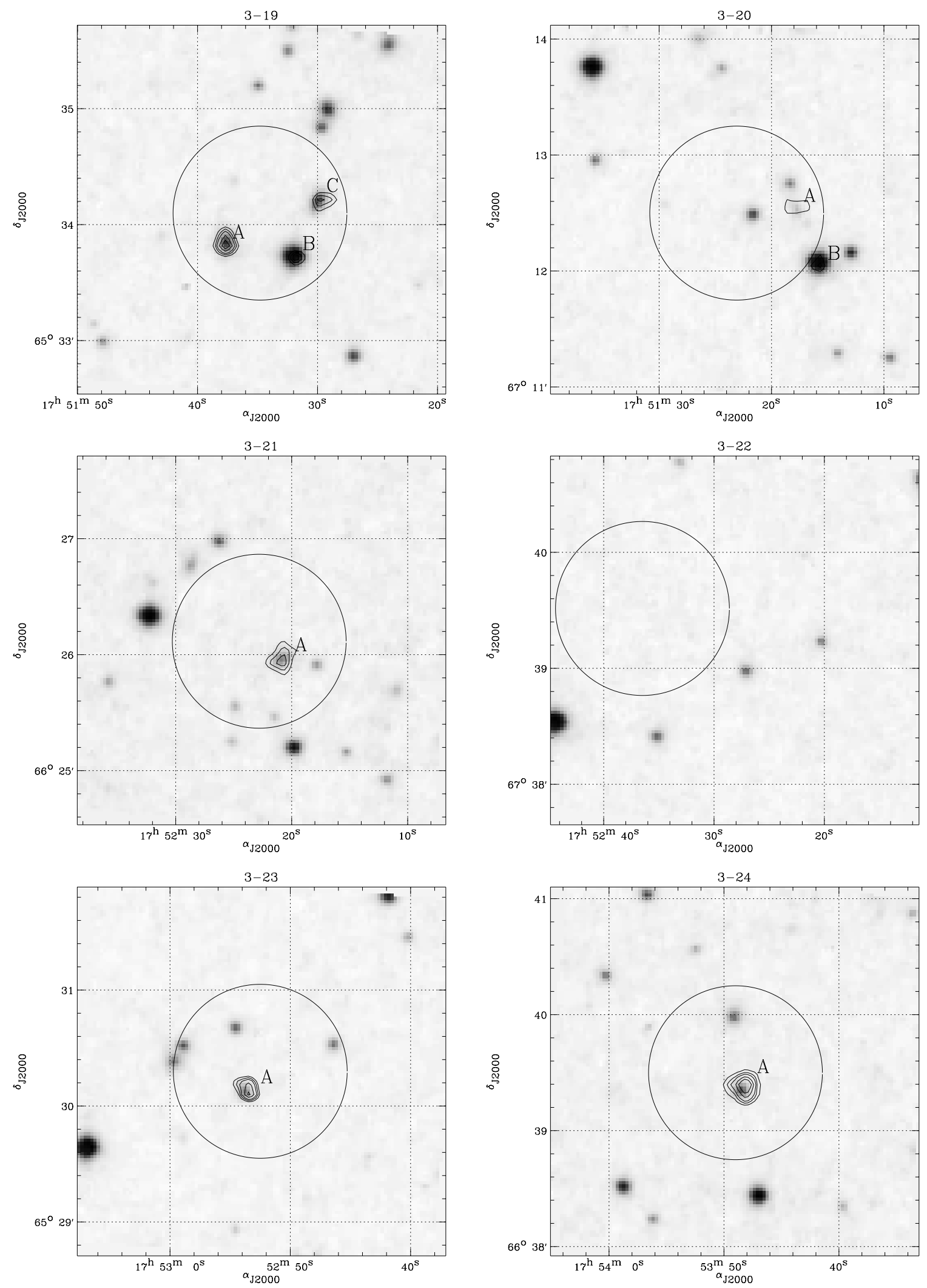

Fig. 4. continued 

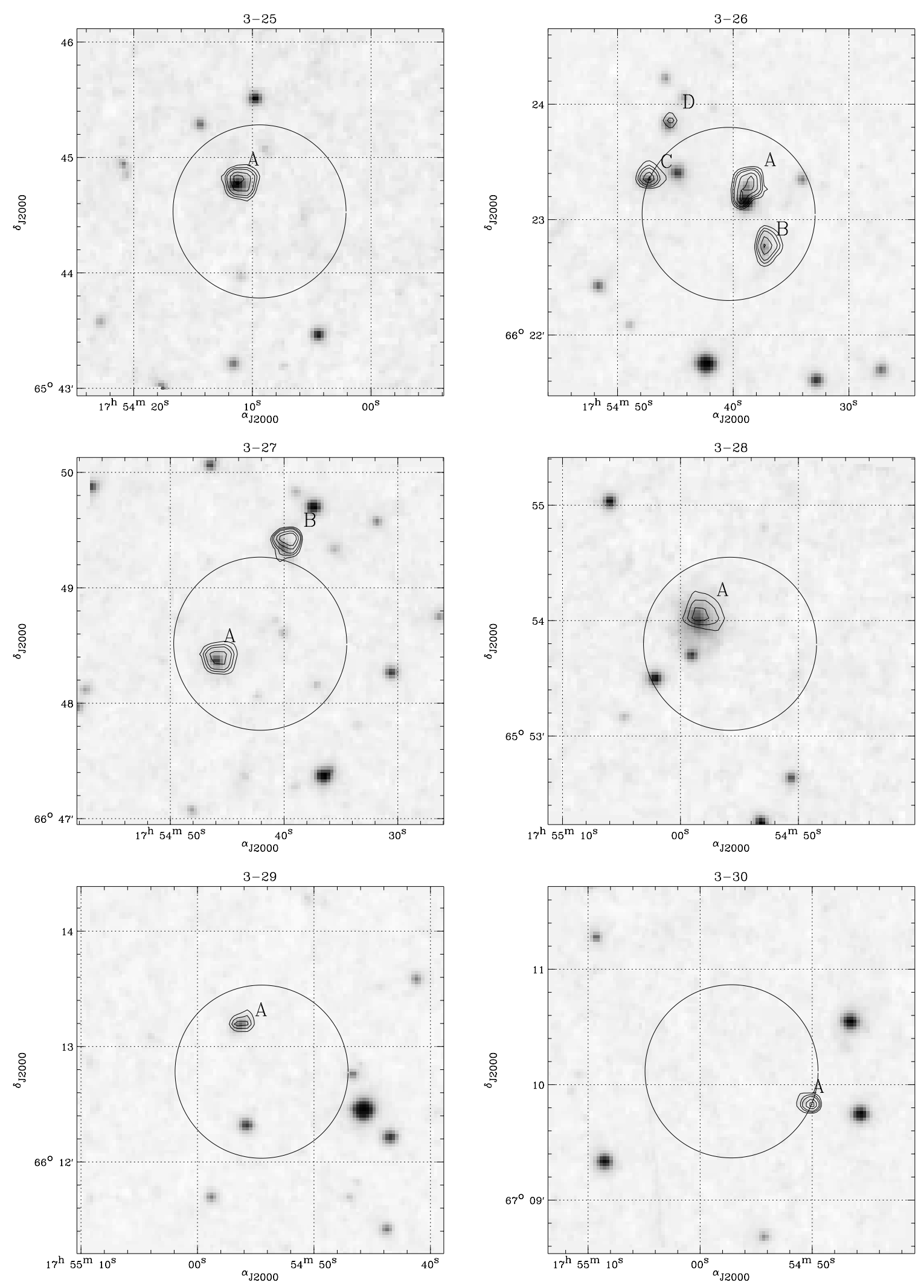

Fig. 4. continued 
H. Aussel et al.: ISOCAM observations of the IRAS NEPR $60 \mu \mathrm{m}$ sample. I.
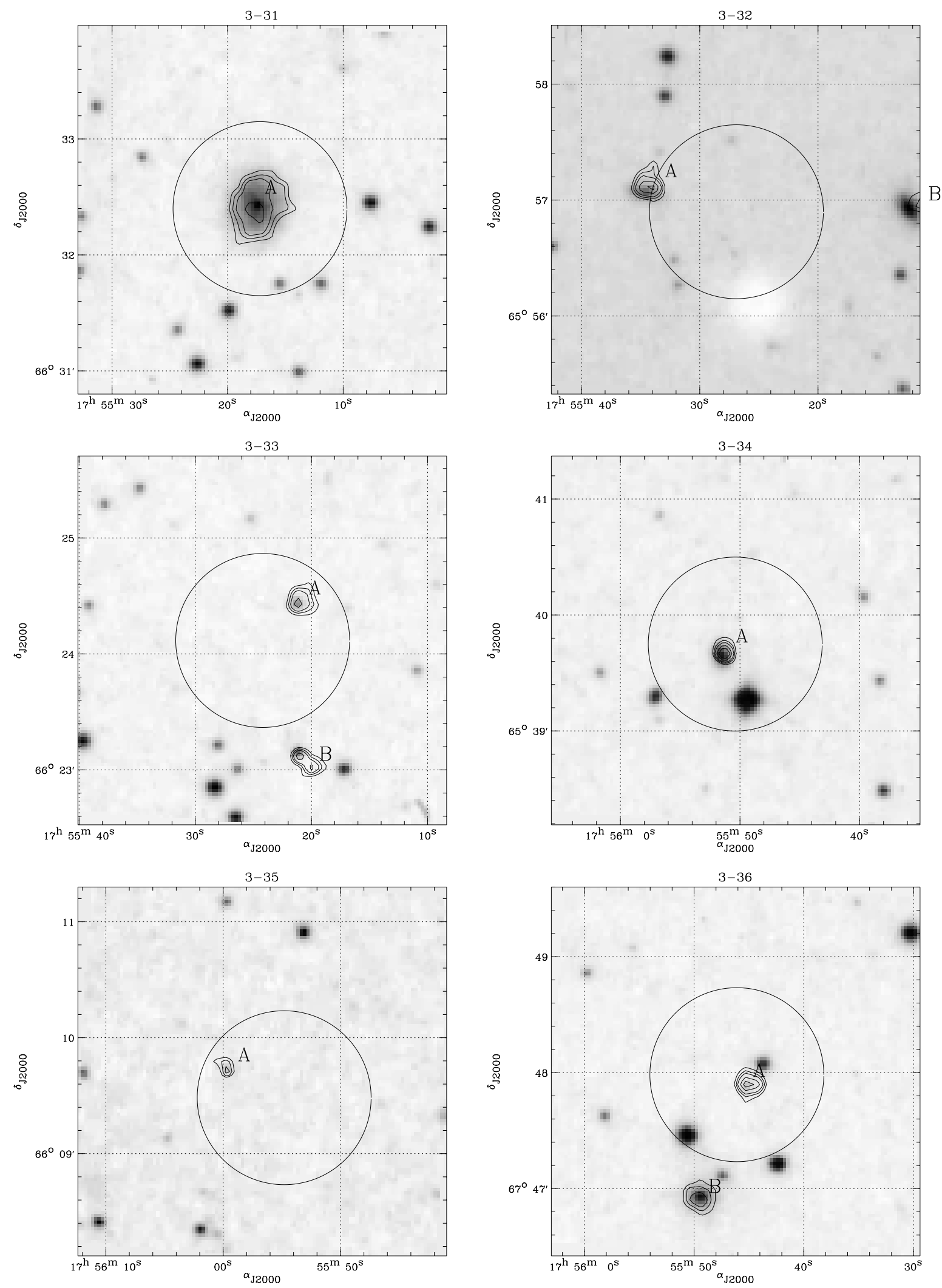

Fig. 4. continued 
H. Aussel et al.: ISOCAM observations of the IRAS NEPR $60 \mu \mathrm{m}$ sample. I.
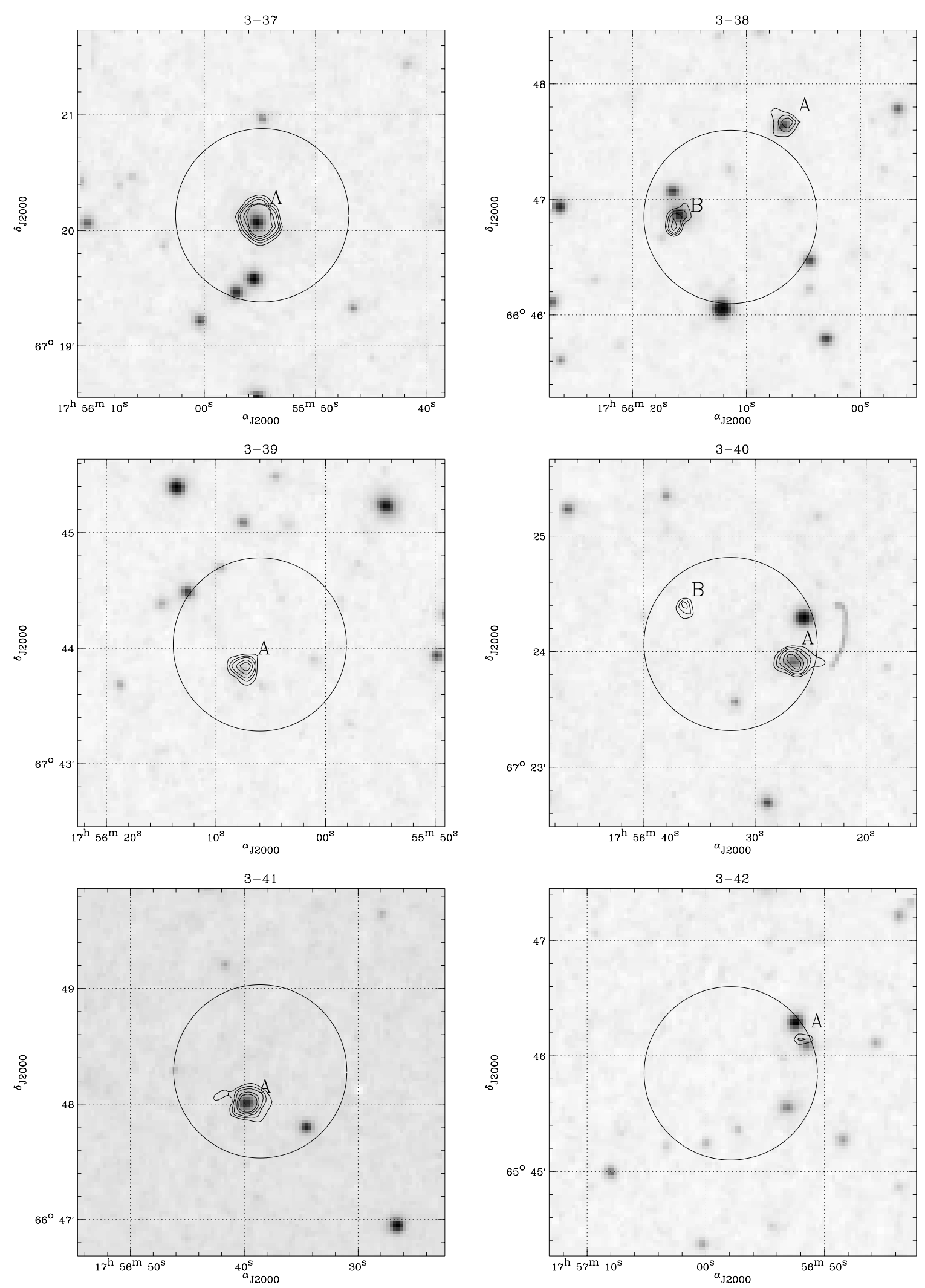

Fig. 4. continued 
H. Aussel et al.: ISOCAM observations of the IRAS NEPR $60 \mu \mathrm{m}$ sample. I.
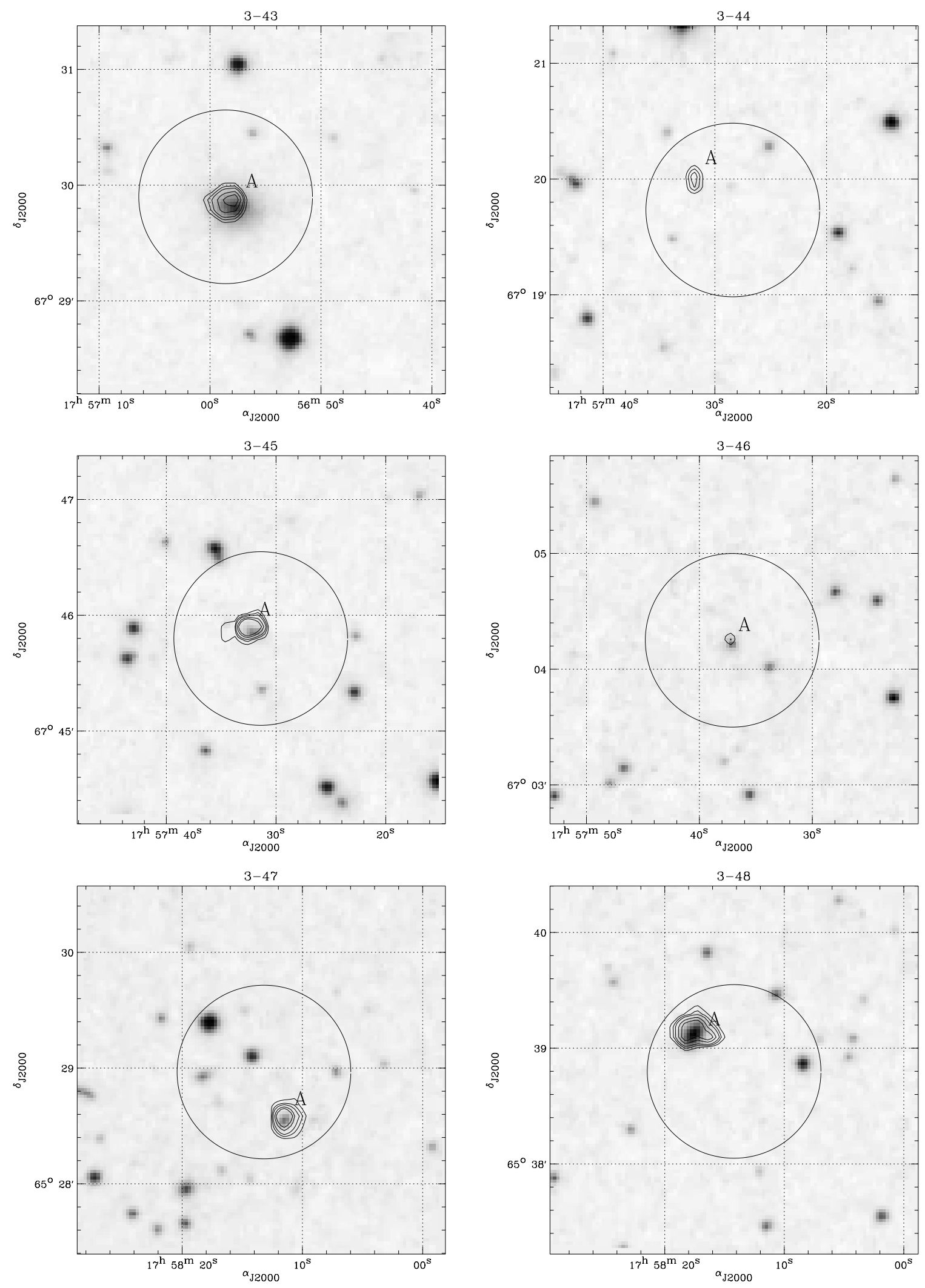

Fig. 4. continued 
H. Aussel et al.: ISOCAM observations of the IRAS NEPR $60 \mu \mathrm{m}$ sample. I.
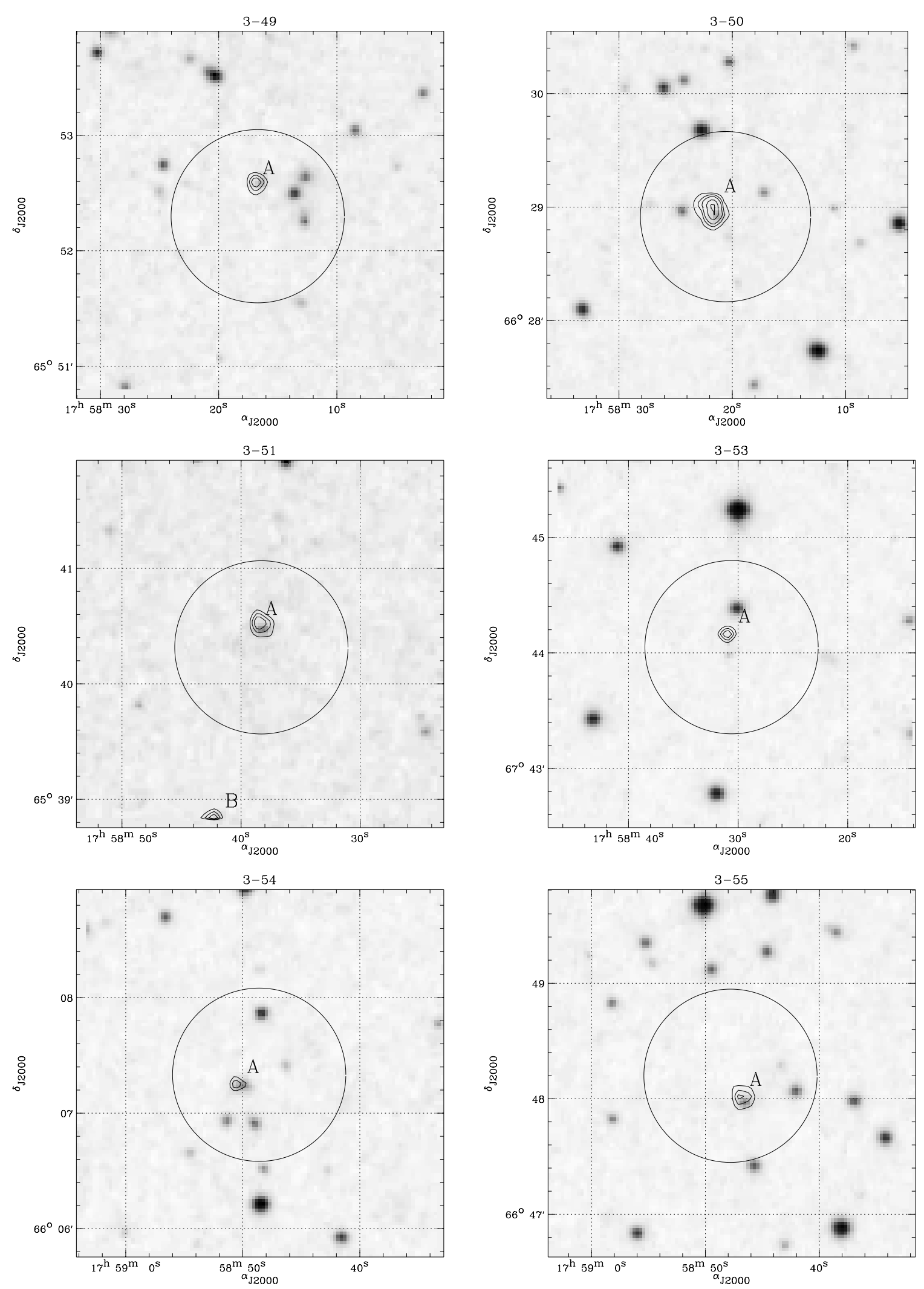

Fig. 4. continued 
H. Aussel et al.: ISOCAM observations of the IRAS NEPR $60 \mu \mathrm{m}$ sample. I.
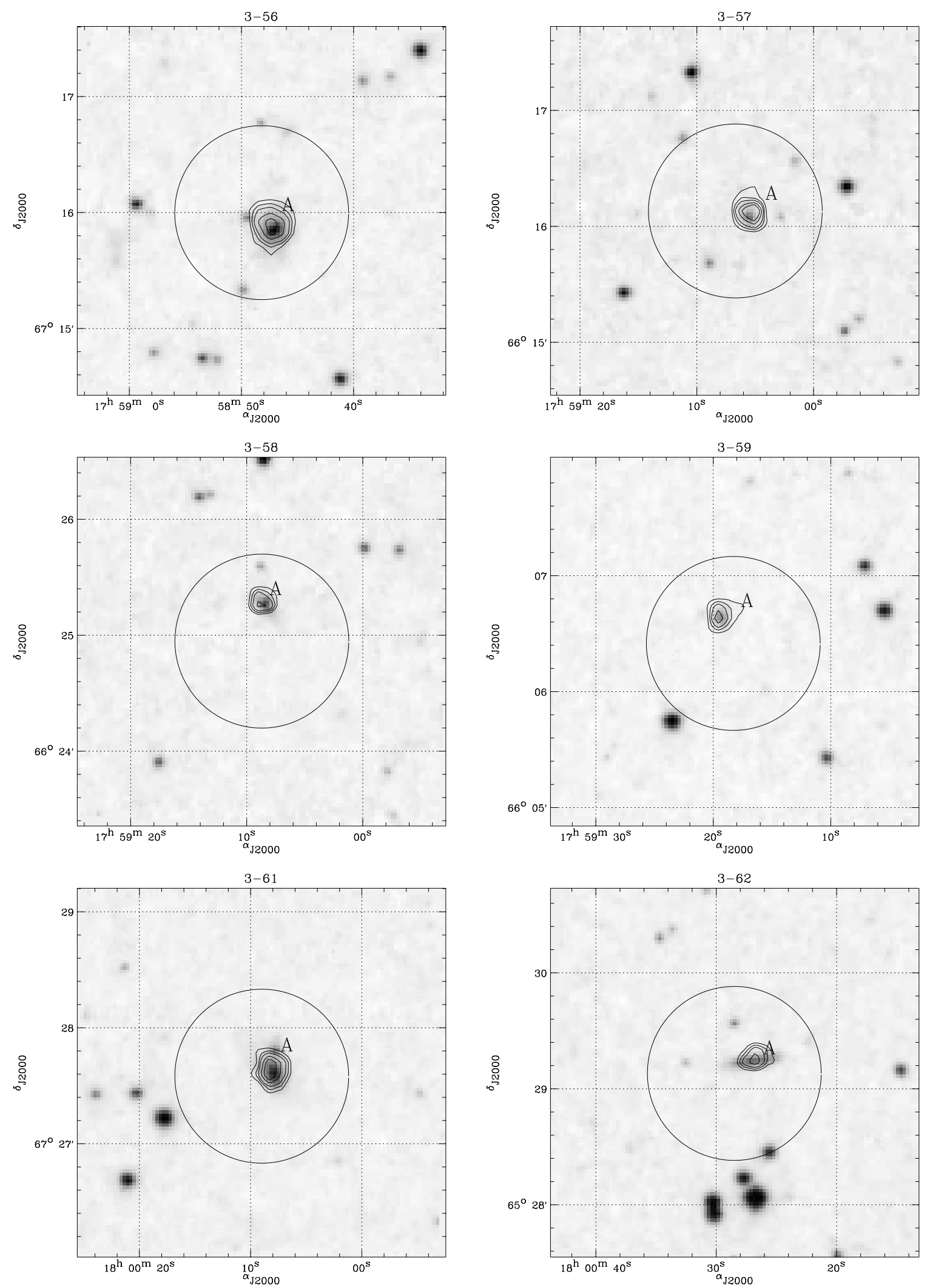

Fig. 4. continued 

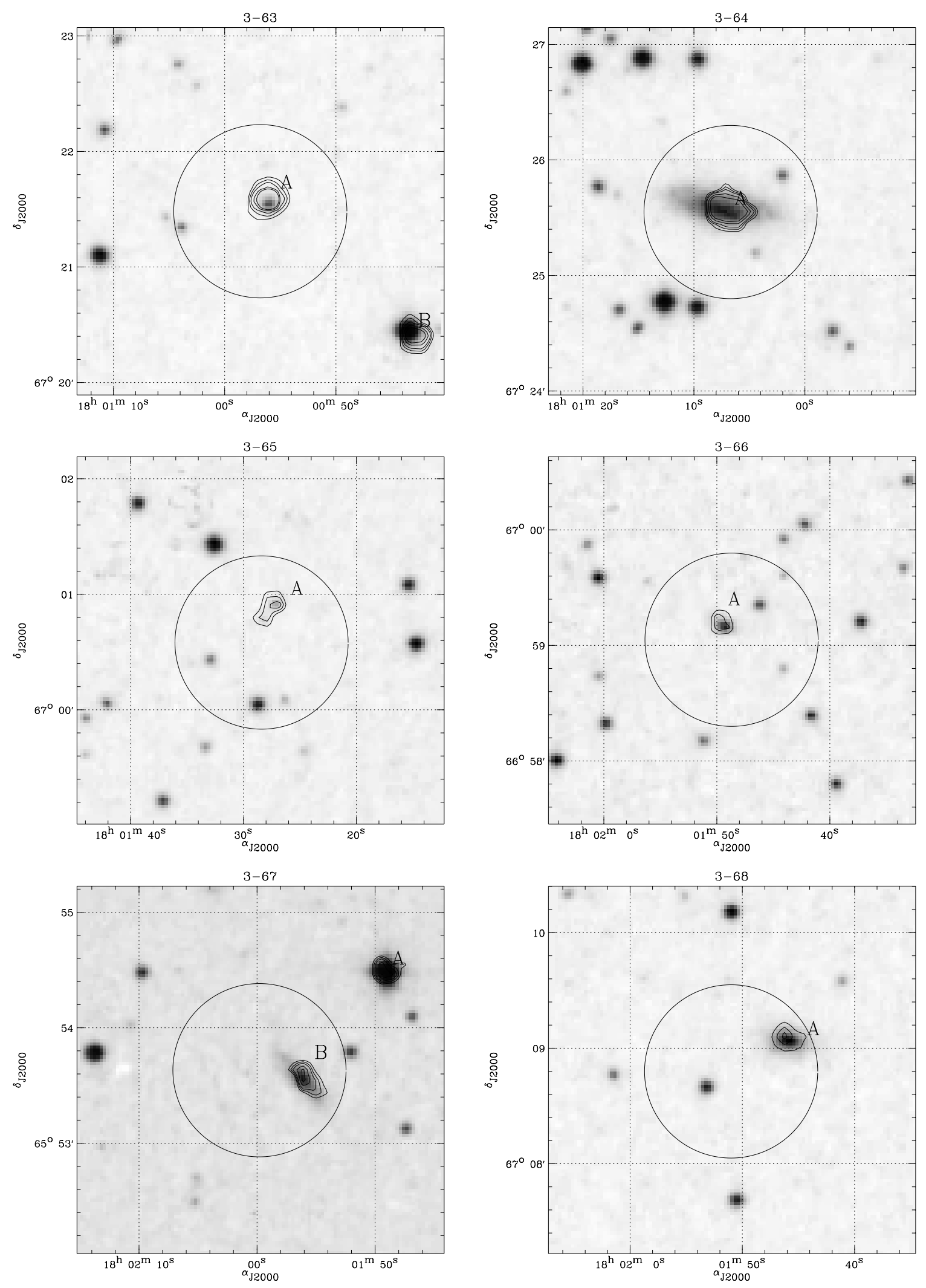

Fig. 4. continued 
H. Aussel et al.: ISOCAM observations of the IRAS NEPR $60 \mu \mathrm{m}$ sample. I.
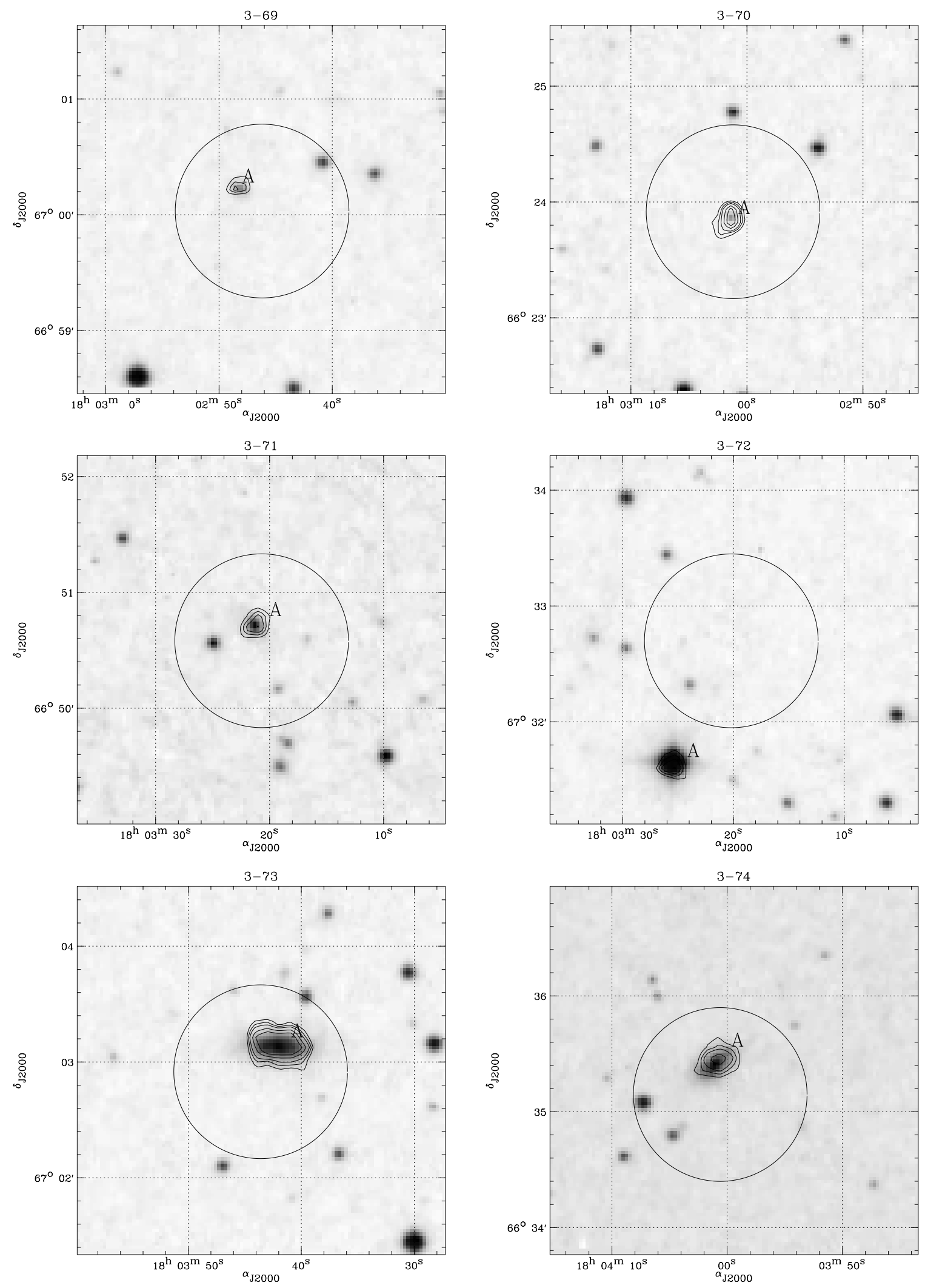

Fig. 4. continued 

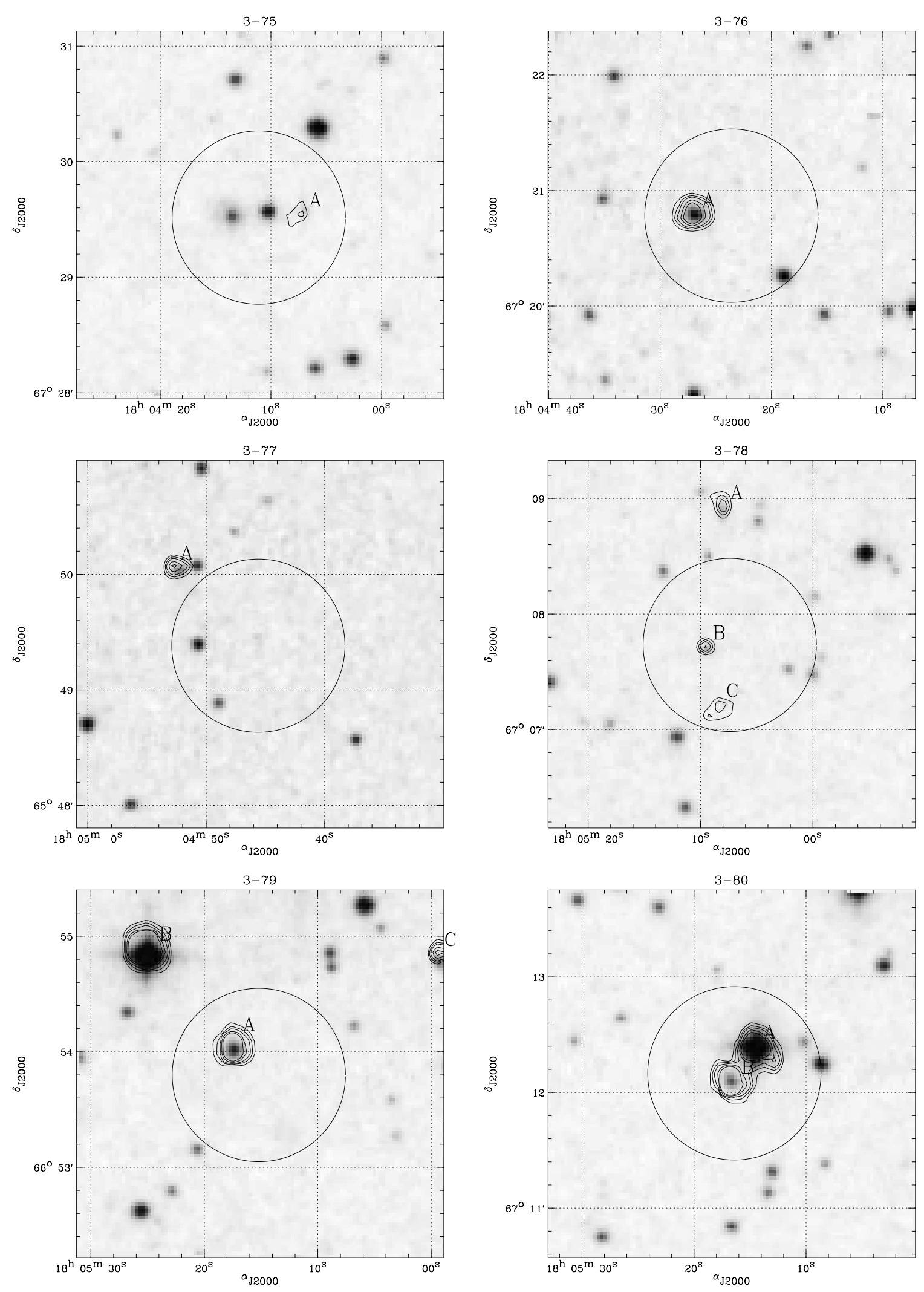

Fig. 4. continued 
H. Aussel et al.: ISOCAM observations of the IRAS NEPR $60 \mu \mathrm{m}$ sample. I.
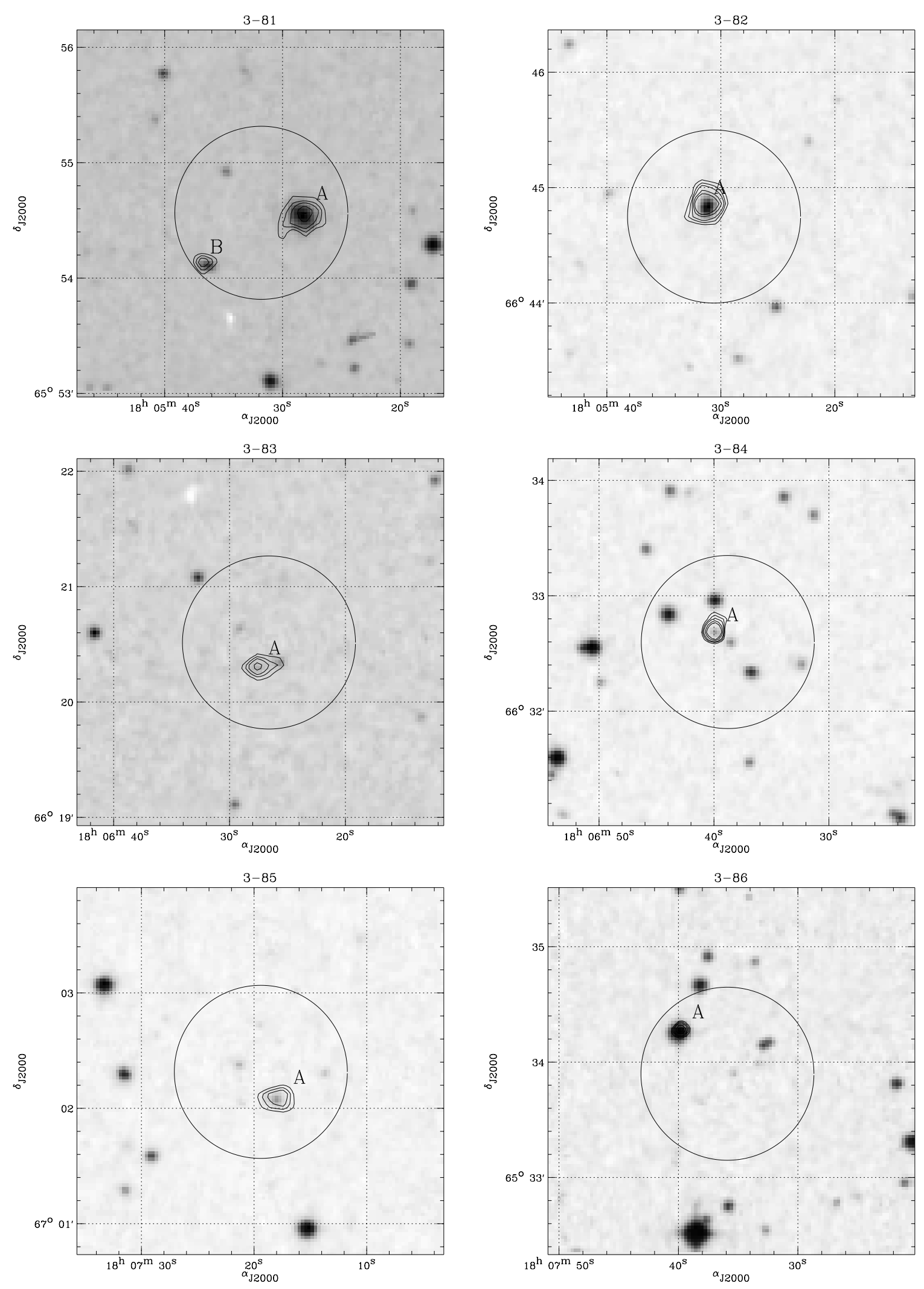

Fig. 4. continued 

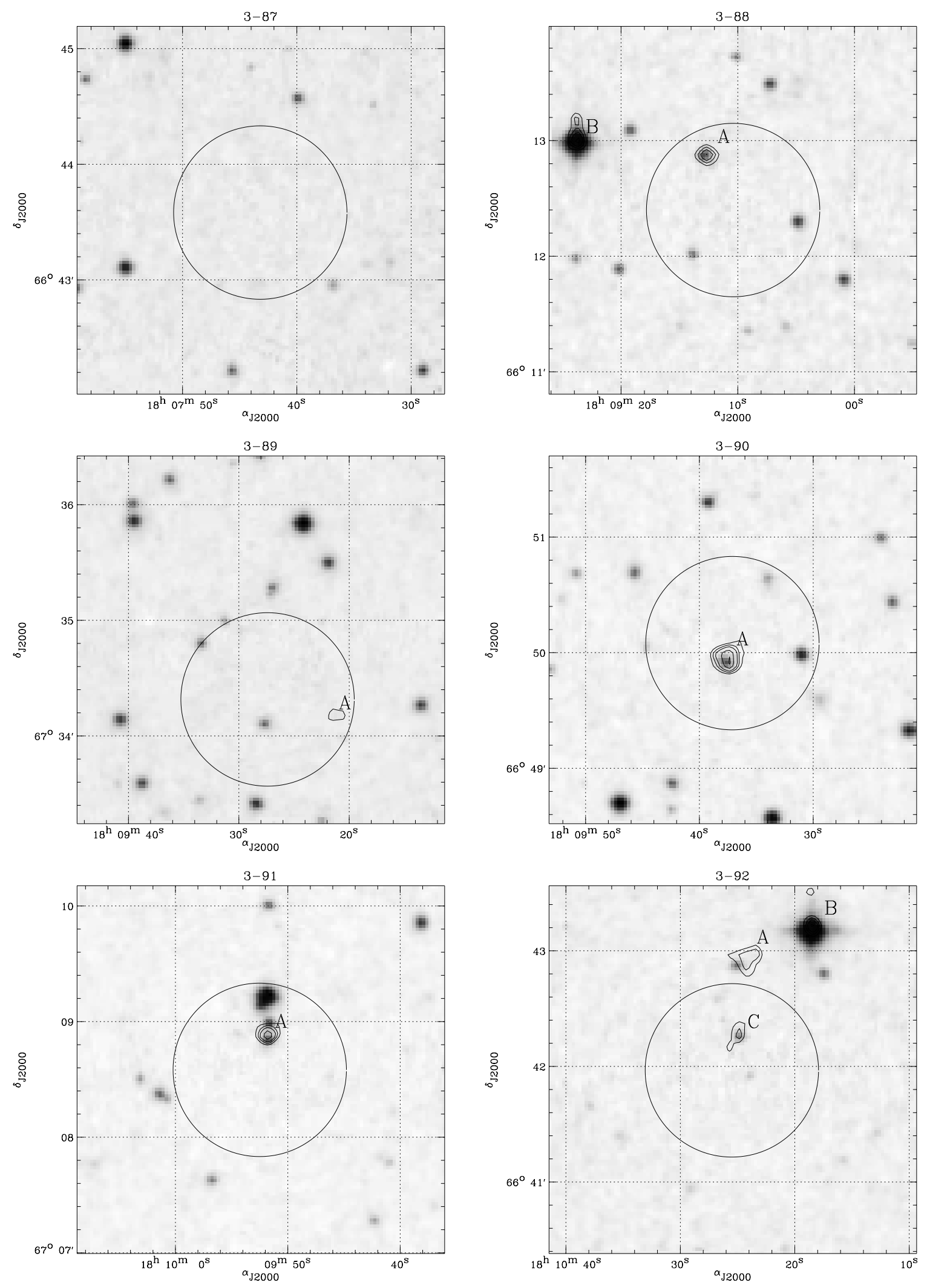

Fig. 4. continued 
H. Aussel et al.: ISOCAM observations of the IRAS NEPR $60 \mu \mathrm{m}$ sample. I.
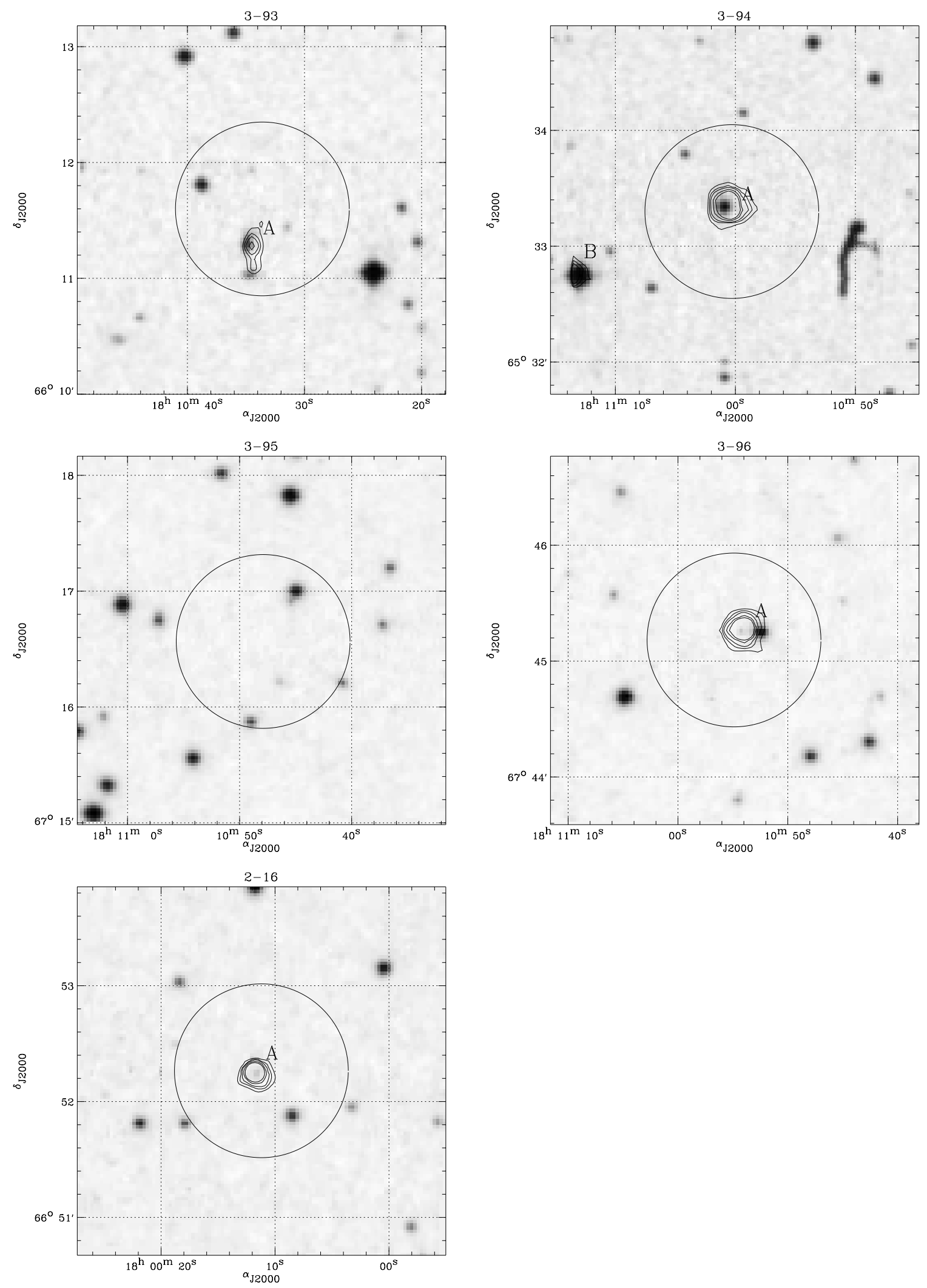

Fig. 4. continued 